\title{
Time-Dependent Aeroelastic Adjoint-Based Aerodynamic Shape Optimization of Helicopter Rotors in Forward Flight
}

\author{
Asitav Mishra* \\ University of Michigan, Ann Arbor, Michigan 48109 \\ Dimitri Mavriplis $\ddagger$ \\ University of Wyoming, Laramie, Wyoming 82071-3295 \\ and \\ Jay Sitaraman \\ Parallel Geometric Algorithms, LLC, Sunnyvale, California 94087 \\ DOI: $10.2514 / 1 . J 054962$
}

\begin{abstract}
A formulation for sensitivity analysis of fully coupled time-dependent aeroelastic rotors in forward flight with trim constraints is given in this paper. The formulation includes the effect of blade shape parameters as well as blade cyclic pitch control parameters to enable analysis and optimization of rotors in a trimmed condition. Both forward sensitivity and adjoint sensitivity formulations are derived that correspond to analogs of the nonlinear aeroelastic analysis problem. Both sensitivity analysis formulations make use of the same iterative disciplinary solution techniques used for analysis, and they make use of an analogous coupling strategy. The fully coupled aeroelastic analysis formulation is first verified to effectively perform performance predictions of a four-bladed rotor in forward flight started impulsively from rest. Upon successful verification of the fully coupled adjoint formulation, it is used to perform trim and aerodynamic shape design optimization for helicopter rotors in forward-flight conditions.
\end{abstract}

\section{Nomenclature}

$A=$ rotor disk area; $\pi R^{2}, \mathrm{~m}^{2}$

$a=$ speed of sound, $\mathrm{m} \cdot \mathrm{s}^{-1}$

$C_{D}=$ two-dimensional drag coefficient

$C_{f}=$ skin-friction coefficient

$C_{L}=$ two-dimensional lift coefficient

$C_{M}=$ two-dimensional pitching moment coefficient

$C_{p}=$ pressure coefficient

$C_{Q}=$ rotor shaft torque coefficient; $Q /\left(\rho A \Omega^{2} R^{3}\right)$

$C_{T}=$ rotor thrust coefficient; $T /\left(\rho A \Omega^{2} R^{2}\right)$

$C d_{0}=$ profile drag coefficient

$c \quad=$ rotor chord, $\mathrm{m}$

$\boldsymbol{D}=$ vector of design variables

$L=$ objective function

$M \quad=$ Mach number; $V / a$

$M_{\text {tip }}=$ rotor tip Mach number; $U_{\text {tip }} / a$

$M_{\infty}=$ freestream Mach number; $U_{\infty} / a$

$R=$ rotor radius, $\mathrm{m}$

$R e=$ Reynolds number; $V c / \nu$

$r \quad=\quad$ radial distance of a rotor spanwise station, $\mathrm{m}$

$U_{\infty}=$ freestream velocity; $\mathrm{ft} \cdot \mathrm{s}^{-1}$

$\boldsymbol{u}=$ flow solution

$V \quad=$ velocity, $\mathrm{m} \cdot \mathrm{s}^{-1}$

$\boldsymbol{x}=$ mesh (interior or surface) coordinates

$\alpha=$ sectional angle of attack, deg

$\alpha_{s}=$ shaft tilt angle, deg

$\Delta t=$ time-step size, deg

Presented as Paper 2015-1130 at the 56th AIAA/ASCE/AHS/ASC Structures, Structural Dynamics, and Materials Conference, Kissimmee, FL, 5-9 January 2015; received 17 December 2015; revision received 14 April 2016; accepted for publication 26 June 2016; published online 30 August 2016. Copyright (C) 2016 by the American Institute of Aeronautics and Astronautics, Inc. All rights reserved. Copies of this paper may be made for personal and internal use, on condition that the copier pay the per-copy fee to the Copyright Clearance Center (CCC). All requests for copying and permission to reprint should be submitted to CCC at www.copyright.com; employ the ISSN 0001-1452 (print) or 1533-385X (online) to initiate your request.

*Postdoctoral Research Scholar, Department of Aerospace Engineering; asitav@gmail.com.

†Professor, Department of Mechanical Engineering; mavrip1@uwyo.edu.

*Research Scientist; jsitaraman@gmail.com.

$$
\begin{array}{ll}
\theta_{0} & =\text { collective pitch, deg } \\
\Lambda & =\text { adjoint solution } \\
\nu & =\text { kinematic viscosity, } \mathrm{m}^{2} \cdot \mathrm{s}^{-1} \\
\rho & =\text { flow density, slugs } \mathrm{m}^{-3} \\
\psi & =\text { azimuth angle, deg } \\
\Omega & =\text { rotor rotational speed, } \mathrm{rad} \cdot \mathrm{s}^{-1}
\end{array}
$$

\section{Introduction}

A DJOINT formulations have become popular for aerodynamic shape-optimization problems using computational fluid dynamics [1-8], largely due to the fact that they allow the computation of sensitivity derivatives of an objective function with respect to a set of given inputs at a cost that is essentially independent of the number of inputs. Although the use of adjoint equations is now fairly well established in steady-state shape optimization, only recently have inroads been made into extending them to unsteady flow problems. A preliminary demonstration of the method's feasibility in three-dimensional problems can be found in [9]. Full implementation in a general sense and application to large-scale problems involving helicopter rotors were carried out by Nielsen et al. in the NASA FUN3D code [10,11], by Lee and Kwon [12], by Mani and Mavriplis [13] using the current flow solver framework, and by a few others from the recent past [14,15].

Since engineering optimization is an inherently multidisciplinary endeavor, the next logical step involves extending adjoint methods to multidisciplinary simulations and using the obtained sensitivities for driving multidisciplinary optimizations. In the context of fixed (and especially rotary-wing) aircraft, aeroelastic coupling effects can be very important and must be considered in the context of a successful optimization strategy. The coupling of computational fluid dynamics (CFD) and computational structural dynamics (CSD), as well as the use of sensitivity analysis on such a system, has been addressed in the past, primarily from a steady-state standpoint $[16,17]$. Until now, relatively little work has been done addressing unsteady aeroelastic optimization problems, mainly due to complexities in the linearization of coupled time-dependent systems. In previous work [18-20], a time-dependent three-dimensional aeroelastic adjoint formulation for helicopter rotor optimization problems was derived. This work built upon a previously demonstrated time-dependent aerodynamic optimization capability that was applied to helicopter rotors in [13], through the addition of a Hodges-Dowell-type [21] beam finite element model to simulate the rotor structure, and the development of the fully coupled discrete adjoint of the resulting 
aeroelastic system. This work demonstrated the effectiveness of using this approach for performing aeroelastic optimization of a representative rotorcraft configuration in hover conditions.

The aim of the current work is to extend the formulation to include time-dependent rotor pitch inputs to accommodate forward-flight analysis and optimization. For shape optimization of rotors in forward flight, pitch inputs not only can feature as design parameters but also can allow for strong rotor trim constraints by making use of force and moment sensitivities with respect to pitch inputs.

In the following sections, we outline the disciplinary components that are used to construct the analysis and adjoint formulations for the flexible rotor problem in forward-flight conditions. These include the flow solver, the finite element beam structural model, a fluidstructure interface, as well as a prescribed blade motion facility and a mesh deformation capability, all described in Sec. II. The general solution procedure of the fully coupled problem at each time step is described in Sec. III. The sensitivity analyses including both the tangent and adjoint formulations are presented in Sec. IV. Unsteady coupled analysis results are discussed, and verification of the adjoint computed sensitivities is then presented in Sec. V. Performance optimization of a helicopter rotor is presented in Sec. VI. Finally, the conclusion section summarizes the results.

\section{Analysis and Sensitivity Formulation}

\section{A. Flow Solver Analysis Formulation}

The flow solver used in this work is the Reynolds-averaged Navier-Stokes unstructured three-dimensional (NSU3D) [22] mesh solver. NSU3D has been widely validated for steady-state and timedependent flows, and it contains a discrete tangent and adjoint sensitivity capability that was demonstrated previously for optimization of steady-state and time-dependent flow problems. As such, only a concise description of these formulations will be given in this paper, with additional details available in previous references $[9,13,22]$. The flow solver is based on the conservative form of the Navier-Stokes equations, which may be written as

$$
\frac{\partial \boldsymbol{u}(\boldsymbol{x}, t)}{\partial t}+\nabla \cdot \boldsymbol{F}(\boldsymbol{u})=0
$$

For moving mesh problems, these are written in arbitrary Lagrangian-Eulerian (ALE) form as

$$
\frac{\partial \boldsymbol{V} \boldsymbol{u}}{\partial t}+\int_{B(t)}[\boldsymbol{F}(\boldsymbol{u})-\dot{\boldsymbol{x}} \boldsymbol{u}] \cdot \boldsymbol{n} \mathrm{d} B=0
$$

Here, $V$ refers to the volume of the control volume bounded by a control surface $B(t), \dot{\boldsymbol{x}}$ is the vector of mesh face or edge velocities, and $\boldsymbol{n}$ is the unit normal of the face or edge. The state vector $\boldsymbol{u}$ consists of the conserved variables, and the Cartesian flux vector $\boldsymbol{F}=$ $\left(\boldsymbol{F}_{x}, \boldsymbol{F}_{y}, \boldsymbol{F}_{z}\right)$ contains both inviscid and viscous fluxes. The equations are closed with the perfect gas equation of state and the SpalartAllmaras turbulent eddy viscosity model [23] for all cases presented in this work. The time derivative term is discretized using a secondorder-accurate backward-difference formula (BDF2) scheme, leading to the implicit system of equations at each time step given as

$$
\frac{3}{2 \Delta t} \boldsymbol{V}^{n} \boldsymbol{u}^{n}-\frac{2}{\Delta t} \boldsymbol{V}^{n-1} \boldsymbol{u}^{n-1}+\frac{1}{2 \Delta t} \boldsymbol{V}^{n-2} \boldsymbol{u}^{n-2}+\boldsymbol{S}^{n}\left(\boldsymbol{u}^{n}, \boldsymbol{x}^{n}, \dot{\boldsymbol{x}}^{n}\right)=0
$$

where $\boldsymbol{V}^{n}=\boldsymbol{V}\left(x^{n}\right)$ represents the mesh control volumes, and $\boldsymbol{S}^{n}\left(\boldsymbol{u}^{n}, \boldsymbol{x}^{n}, \dot{\boldsymbol{x}}^{n}\right)$ represents the spatial discretization terms at the $n$th time step. The functional dependence of the implicit system to be solved at each time step can be written in residual form as

$$
\boldsymbol{R}^{n}\left(\boldsymbol{u}^{n}, \boldsymbol{u}^{n-1}, \boldsymbol{u}^{n-2}, \boldsymbol{x}^{n}, \boldsymbol{x}^{n-1}, \boldsymbol{x}^{n-2}\right)=0, \quad n=, 2,3, \ldots, N
$$

where the initial conditions are given by $\boldsymbol{u}^{o}$ and $\boldsymbol{x}^{o}$, and noting that a first-order-accurate backward-difference formula time discretization is used for the first time step.

At each time step, the implicit residual is solved using Newton's method. The Jacobian matrix is inverted iteratively using a line- implicit agglomeration multigrid scheme that can also be used as a preconditioner for a Generalized Minimum RESidual (GMRES) Krylov solver [24]. To simplify the notation, we drop the time-level superscripts and represent the general flow equations to be solved at all time levels as the system given by

$$
\boldsymbol{R}(\boldsymbol{u}, \boldsymbol{x})=0
$$

where the vector $\boldsymbol{U}$ denotes the flow values over all time steps, $\boldsymbol{x}$ represents the CFD mesh coordinates over all time steps, and Eq. (5) denotes the simultaneous solution of all time steps. Given the functional dependence of Eq. (4), it is evident that Eq. (5), when written in matrix form, takes on a lower triangular form and can be solved by forward substitution, which corresponds to a forward integration in time.

\section{B. Structural Model Formulation}

A nonlinear bend-twist beam model is a suitable and widely used structural model for slender fixed- and rotary-wing aircraft structures within the context of an aeroelastic problem. A bend-twist beam model was previously developed and coupled to the NSU3D unstructured mesh Reynolds-averaged Navier-Stokes solver for steady [25,26] and time-dependent problems [20]. The nonlinear governing equations of a slender beam are discretized using the finite element method in space using Hamilton's principle, as described in detail in the work by Hodges and Dowell [21]. Figure 1 shows a typical beam with $15 \mathrm{deg}$ of freedom for each element to accommodate bendwise, lagwise, axial, and torsional displacements. The elastic twist $\phi$ refers to the rotation about the blade elastic axis. The second-order equation of motion for the beam can be expressed as $M \ddot{\boldsymbol{q}}+C \dot{\boldsymbol{q}}+K \boldsymbol{q}=F$, where $[M],[C]$, and $[K]$ are the mass, damping, and stiffness matrices of the system of equations representing the beam. Vector $F=F(t)$ is the forcing vector. Vector $\boldsymbol{q}$ represents the displacements along all degrees of freedom. This set of equations can be reduced to a first-order system as $[I] \dot{\boldsymbol{Q}}+[A] \boldsymbol{Q}=\boldsymbol{F}$, where $[I]$ is the identity matrix, $\boldsymbol{Q}=[\boldsymbol{q}, \dot{\boldsymbol{q}}]^{T}, \boldsymbol{F}=\left[0,[M]^{-1} \mathrm{~F}\right]^{T}$, and

$$
[A]=\left[\begin{array}{cc}
0 & -[I] \\
{[M]^{-1}[K]} & {[M]^{-1}[C]}
\end{array}\right]
$$

Similar to the flow equations, a second-order backward-difference time-integration scheme (BDF2) is used to advance the structural equations in time. This results in the system of equations to be solved at each time step are given as

$$
\frac{3}{2 \Delta t} \boldsymbol{Q}^{n}-\frac{2}{\Delta t} \boldsymbol{Q}^{n-1}+\frac{1}{2 \Delta t} \boldsymbol{Q}^{n-2}+[A]^{n} \boldsymbol{Q}^{n}=\boldsymbol{F}^{n}
$$

The functional dependence of the structural dynamic equations to be solved at each time step can be written in residual form as

$$
\boldsymbol{J}\left(\boldsymbol{Q}^{n}, \boldsymbol{Q}^{n-1}, \boldsymbol{Q}^{n-2}, \boldsymbol{F}^{n}\right)=0, \quad n=, 2,3, \ldots, N
$$

As with the flow equations, to simplify the notation, we drop the time-level superscripts and represent the structural dynamic equations to be solved at all time levels as the system given by

$$
J(Q, F)=0
$$

where $\boldsymbol{Q}$ and $\boldsymbol{F}$ represent the structural states and forces, respectively, over all time steps. Written in matrix form, Eq. (8) exhibits a lower triangular form and can be solved by forward substitution in time, which corresponds to a forward time-integration procedure. The beam model has been validated [27] for the standard HART-II rotor case [28] by comparing its natural frequency predictions with the predictions from other reliable CSD models, such as UMARC [29] and DLR [30].

\section{Forward Sensitivity Formulation of Beam Model}

The beam tangent (forward sensitivity) linearization is similar to the analysis problem. For a given functional $L(D, Q(D))$, its sensitivity with respect to a blade design parameter $\boldsymbol{D}$ can be written as 


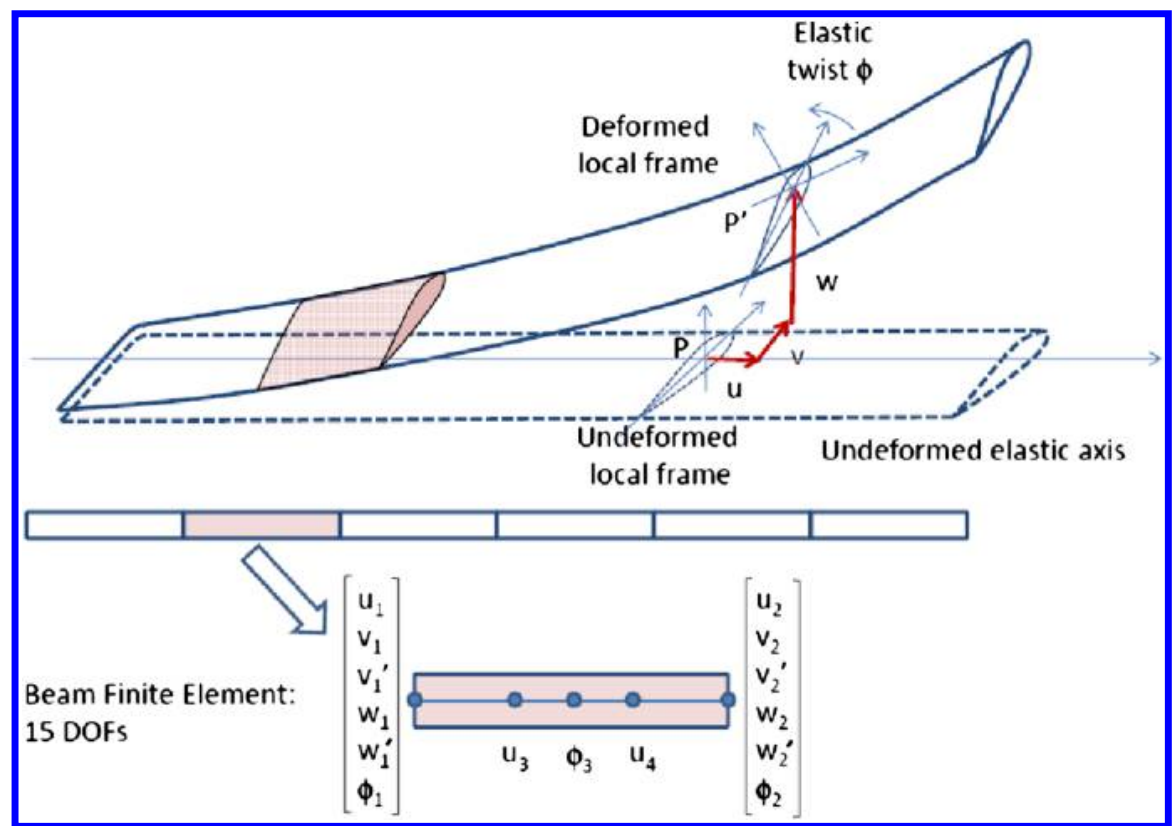

Fig. 1 Beam element with flap, lag, torsional, and axial (total 15) degrees of freedom (DOFs).

$$
\frac{\mathrm{d} L}{\mathrm{~d} \boldsymbol{D}}=\frac{\partial L}{\partial \boldsymbol{D}}+\frac{\partial L}{\partial \boldsymbol{Q}} \frac{\mathrm{d} \boldsymbol{Q}}{\mathrm{d} \boldsymbol{D}}
$$

This requires solving for sensitivity of the beam state $Q$, which can be obtained by differentiating Eq. ()ㅡ with respect to the design variable $\boldsymbol{D}$ and rearranging as

$$
\left[\frac{\partial \boldsymbol{J}}{\partial \boldsymbol{Q}}\right] \frac{\partial \boldsymbol{Q}}{\partial \boldsymbol{D}}=-\frac{\partial \boldsymbol{J}}{\partial \boldsymbol{F}} \frac{\mathrm{d} \boldsymbol{D}}{\mathrm{D}}-\frac{\partial \boldsymbol{J}}{\partial \boldsymbol{D}}
$$

Solving for $\partial \boldsymbol{Q} / \partial \boldsymbol{D}$ in Eq. (10), and substituting this into Eq. (9), the forward sensitivity of the objective function $\mathrm{d} L / \mathrm{d} \boldsymbol{D}$ can be obtained.

\section{Adjoint Formulation of Beam Model}

The adjoint formulation of the beam model can be derived by approaching the tangent formulation in the reverse (transpose) direction. Taking the transpose of the objective functional sensitivity yields

$$
\frac{\mathrm{d} L^{T}}{\mathrm{~d} \boldsymbol{D}}=\frac{\partial L^{T}}{\partial \boldsymbol{D}}+\frac{\mathrm{d} \boldsymbol{Q}}{\mathrm{d} \boldsymbol{D}} \frac{\partial L^{T}}{\partial \boldsymbol{Q}}
$$

This requires solving for the transpose sensitivity of the beam state $Q$, which leads to solving for an adjoint vector $\Lambda_{Q}$ defined as

$$
\left[\frac{\partial \boldsymbol{J}}{\partial \boldsymbol{Q}}\right]^{T} \Lambda_{\boldsymbol{Q}}=\frac{\partial L^{T}}{\partial \boldsymbol{Q}}
$$

The final objective sensitivities can be obtained by

$$
{\frac{\mathrm{d} L^{T}}{\mathrm{~d} \boldsymbol{D}}}^{T}{\frac{\partial L^{T}}{\partial \boldsymbol{D}}}^{T}+\left[-\frac{\partial \boldsymbol{J}}{\partial \boldsymbol{D}}^{T}-\frac{\mathrm{d}}{\mathrm{F} \boldsymbol{D}}^{T} \frac{\partial \boldsymbol{J}^{T}}{\partial \boldsymbol{F}}\right] \Lambda_{\boldsymbol{Q}}
$$

The preceding forms the adjoint formulation of the beam model. In this work, we consider only shape design variables that affect the outer mold line of the CFD geometry without modifying the structural model. Thus, the influence of these aerodynamic design variables is only felt through changes in the aerodynamic forces applied to the structural model, which correspond to the second term in the brackets on the righthand side of the preceding equation. The first term in the brackets vanishes in this case, but it would be nonzero when considering structural design parameters such as material properties. As previously, the left-hand-side Jacobian term of the structural adjoint equation corresponds to the transpose of the Jacobian in the forward linearization. In earlier work by Mishra et al. [18], forward and adjoint formulations of the beam solver were implemented and verified for both structural design parameters (such as element stiffnesses elastic modulus or Young's modulus (E) moment of inertia (I), rigidity modulus (G) torsion constant (J), and element masses) and force-based design parameters (as required for the coupled aeroelasticity problem).

\section{Fluid-Structure Interface}

In addition to the solution of the aerodynamic problem and the structural dynamics problem, the solution of the fully coupled timedependent aeroelastic problem requires the exchange of aerodynamic loads from the CFD solver to the beam structure, which in turn returns surface displacements to the fluid flow solver. In practice, the fluidstructure interface (FSI) computes forces at each CFD surface mesh point $\boldsymbol{F}(\boldsymbol{x}, \boldsymbol{u})$ by integrating the surface stresses (pressure and shear) over the surface area associated with each surface grid point. These forces are then projected onto the beam finite element basis functions where they are assembled in the form of forces on the beam finite element nodal locations denoted as $\boldsymbol{F}_{b}$. Conversely, once the beam deflections have been computed, the structural displacements are transferred back to the surface CFD mesh in a similar manner. The governing equations for the FSI can be written in residual form as

$$
\begin{gathered}
\boldsymbol{S}^{\mathrm{FSI}}\left(\boldsymbol{F}_{b}, \boldsymbol{x}, \boldsymbol{u}\right)=\boldsymbol{F}_{b}-[T(\boldsymbol{Q})] \boldsymbol{F}(\boldsymbol{x}, \boldsymbol{u})=0 \\
\boldsymbol{S}^{\mathrm{SFI}}\left(\boldsymbol{x}_{s}, \boldsymbol{Q}\right)=\boldsymbol{x}_{s}-[T(\boldsymbol{Q})]^{T} \boldsymbol{Q}=0
\end{gathered}
$$

where $\boldsymbol{S}^{\mathrm{FSI}}$ represents the force transfer from CFD to CSD, and $\boldsymbol{S}^{\mathrm{SFI}}$ denotes the displacement transfer from CSD to CFD. In these equations, $[T]$ represents the rectangular transfer matrix that projects pointwise CFD surface forces $\boldsymbol{F}(\boldsymbol{x}, \boldsymbol{u})$ onto the individual beam elements, resulting in the beam forces $\boldsymbol{F}_{b}$. The transpose of this matrix is used to obtain the CFD surface displacements $\boldsymbol{x}_{s}$ from the beam degrees of freedom $\boldsymbol{Q}$, thus ensuring the principle of conservation of virtual work. Also note that $[T]$ is a function of $\boldsymbol{Q}$, since the transfer patterns change with the beam deflection.

\section{Prescribed Motion Facility}

The prescribed motion of the rotor blades must be applied in order to simulate a rotor in forward flight. For a fixed hub, the overall rotor motion can be broken down into two parts: the cyclic pitching of the blades defined by a pitch angle $\theta$, and the azimuthal rotation of the blades about the hub axis $\psi$, as shown in Fig. 2. The time-varying blade pitch angle can be represented by a combination of mean pitch angle $\theta_{0}$ and several harmonic components of pitch angles (e.g., $\theta_{c_{i}}$ and $\theta_{s_{i}}$ for the $i$ th harmonic): $\theta=\theta_{0}+\theta_{c_{i}} \cos (i \psi)+\theta_{s_{i}} \sin (i \psi)$, for harmonics of $i=[1,2, \cdots]$. This introduces the set of control parameters (i.e., 
$\left.\boldsymbol{D}=\left[\theta_{0}, \theta_{c_{i}}, \theta_{s_{i}}\right]\right)$, which define the prescribed blade cyclic pitching motion. In this work, three pitch parameters are used, namely, collective $\theta_{0}$ and two cyclics $\left(\theta_{c_{1}}, \theta_{s_{1}}\right)$ corresponding to a single harmonic $i=1$. At each time step, the individual blade pitch angles are computed using the aforementioned control inputs and used to displace all CFD surface mesh points by the required angular rotation $\theta$. Thus, the pitching motion results in a new set of surface grid points defined by the solution of the blade pitching equation in residual form:

$$
\boldsymbol{S}^{\theta}\left(\boldsymbol{x}_{\mathrm{sp}}, \boldsymbol{x}_{s}, \boldsymbol{D}\right)=0
$$

where $\boldsymbol{x}_{\mathrm{sp}}$ represents the new pitched blade surface mesh points; $\boldsymbol{x}_{s}$ represents the original surface mesh points; and $\boldsymbol{D}$ represents the control inputs, which may also be used as design parameters for optimization runs.

Once the surface mesh points have been displaced, the interior mesh must be deformed to avoid negative cells in neighboring regions. This is accomplished using the mesh deformation facility described subsequently in Sec. II.F, resulting in a new set of interior mesh points denoted as $\boldsymbol{x}_{o}$. The azimuthal rotation is next applied by rotating the entire grid as a solid body. This is achieved by solving the prescribed mesh rotation equations, written in residual form as

$$
S^{\psi}\left(x, x_{o}\right)=0
$$

where $\boldsymbol{x}$ denotes the final deformed and rotated mesh coordinates.

\section{E. Geometry Parametrization Facility}

To perform shape optimization of a multibladed rotor, a shape parametrization capability must be implemented. The specific pa rametrization used in this work is described in Sec. V.B.1. Mathematically, the blade design methodology defines a blade surface, which in turn defines the coordinates of the CFD surface mesh points on each blade in a fixed reference frame as a function of a set of shape design variables $\boldsymbol{D}$. Thus, the blade surface mesh points $\boldsymbol{x}_{\text {so }}$ are obtained as the solution of the parametrization equations in residual form:

$$
\boldsymbol{B}_{\text {cad }}\left(\boldsymbol{x}_{\mathrm{so}}, \boldsymbol{D}\right)=0
$$

where $\boldsymbol{B}_{\text {cad }}$ denotes a possible computer-aided design (CAD) parametrization of the blade, and $\boldsymbol{D}$ refers to the blade shape parameters, such as Hicks-Henne bump functions, blade twist, and pitching parameters described in Sec. V.B.1.

\section{F. Mesh Deformation Capability}

A mesh deformation capability is required to deform the volume mesh in response to prescribed displacements of the surface CFD mesh. Surface displacements can be produced in three manners: 1) through blade geometry shape changes resulting during the design process; 2) through blade deflections generated by the structural model; and 3) due to the prescribed blade cyclic pitching, defined by the three pitch control parameters. In practice, a single mesh deformation calculation is performed at each coupling cycle. Thus, the surface displacements are first determined by adding together the

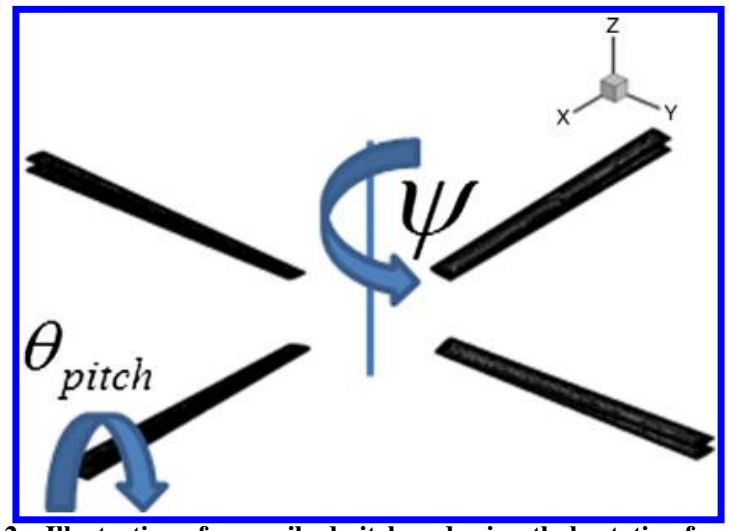

Fig. 2 Illustration of prescribed pitch and azimuthal rotation for rotor in forward flight. displacements from the three sources when present, and the interior mesh point coordinates are obtained by solving the mesh deformation equations, which can be written in residual form as

$$
\boldsymbol{G}\left(\boldsymbol{x}_{o}, \boldsymbol{x}_{\mathrm{sp}}\right)=0
$$

where $\boldsymbol{x}_{o}$ denotes the deformed interior mesh coordinates in response to the displaced surface mesh coordinates $\boldsymbol{x}_{\mathrm{sp}}$. It should be mentioned here that the structural effects of pitching accelerations have been ignored owing to relatively higher torsional rigidity, and consequently lower torsional natural frequencies of HART-II rotor. Note that the mesh deformation calculation is carried out before the azimuthal mesh rotation phase, which operates on the deformed coordinates $\boldsymbol{x}_{o}$ before rotation to produce the final mesh coordinates $\boldsymbol{x}$ used in the CFD simulation, as described by Eq. (16). The mesh deformation approach is based on a linear elastic analogy. In this approach, the mesh is modeled as a linear elastic solid with a variable modulus of elasticity that can be prescribed either as inversely proportional to cell volume or to the distance of each cell from the nearest wall (and thus is a function of current grid). The resulting equations are discretized and solved on the mesh in its original undeformed configuration in response to surface displacements using a line-implicit multigrid algorithm analogous to that used for the flow equations. A detailed explanation of the method can be found in $[25,31]$. An equivalent adjoint solver [31] for the mesh deformation problem is also implemented for use in the coupled aeroelastic adjoint capability.

\section{General Solution Procedure}

The general analysis problem consists of multiple coupled sets of equations, namely, the blade design equations, the prescribed motion equations, the mesh deformation equations, the flow solution equations, the structural model equations, and the fluid-structure interface. All these equations are coupled and must be solved simultaneously at each time step. The coupled system of equations to be solved can be written as

$$
\begin{gathered}
\boldsymbol{B}_{\mathrm{cad}}\left(\boldsymbol{x}_{\mathrm{so}}, \boldsymbol{D}\right)=0 \\
\boldsymbol{S}^{\theta}\left(\boldsymbol{x}_{\mathrm{sp}}, \boldsymbol{x}_{\mathrm{so}}, \boldsymbol{x}_{s}, \boldsymbol{D}\right)=0 \\
\boldsymbol{G}\left(\boldsymbol{x}_{o}, \boldsymbol{x}_{\mathrm{sp}}\right)=0 \\
\boldsymbol{S}^{\psi}\left(\boldsymbol{x}, \boldsymbol{x}_{o}\right)=0 \\
\boldsymbol{R}(\boldsymbol{u}, \boldsymbol{x})=0 \\
\boldsymbol{S}^{\mathrm{FSI}}\left(\boldsymbol{F}_{b}, \boldsymbol{x}, \boldsymbol{u}\right)=0 \\
\boldsymbol{J}\left(\boldsymbol{F}_{b}, \boldsymbol{Q}\right)=0 \\
\boldsymbol{S}^{\mathrm{SFI}}\left(\boldsymbol{x}_{s}, \boldsymbol{Q}\right)=0
\end{gathered}
$$

In Eq. (20), the cyclic pitching acts directly on the deflected blade shape coordinates $\boldsymbol{x}_{s}$, although these depend on the original undeflected blade surface coordinates $\boldsymbol{x}_{\text {so }}$ and reduce to these values at the beginning of the solution procedure before the solution of the structural equations. The preceding equations denote the system of equations to be solved over all time steps. However, since each residual equation at a given time level $n$ depends on (at most) the values from time levels $n, n-1$, and $n-2$, the entire system can be solved by forward substitution in time (i.e., forward time integration). In this case, the equations to be solved at a given time level $n$ can be written more precisely by reintroducing the time-level superscripts on the residual operators and their corresponding arguments. 
Within each physical time step, the solution of the fully coupled fluid-structure problem consists of performing multiple coupling iterations on each discipline using the latest available values from the other disciplines. Thus, a typical solution procedure starts by generating the blade surface mesh coordinates and prescribing the required pitch angles to these points, as determined by the first two equations, which can be evaluated explicitly. Next, the mesh deformation equations [i.e., Eq. (21)] must be solved iteratively, after which the deformed mesh is rotated through explicit evaluation of Eq. (22). The flow equations [i.e., Eq. (23)] can then be solved iteratively using the updated grid coordinates $\boldsymbol{x}$, and the resulting flow variables $\boldsymbol{u}$ are then used to compute forces which are applied to the structural model through explicit evaluation of the FSI [i.e., Eq. (24)]. Using these forces, the structural model is solved directly [i.e., Eq. (25)] and the resulting displacements are transferred back to the CFD mesh as determined by Eq. (26). Since the surface mesh coordinates are now modified, the entire process must be repeated, starting at the second equation, until convergence is achieved.

\section{Sensitivity Analysis for Coupled Aeroelastic Problem}

In this work, we consider only aerodynamic performance objectives that depend only on the flow solution and geometric shape. Starting with the forward sensitivity problem, the sensitivity of such an objective $L$ can be written as

$$
\frac{\mathrm{d} L}{\mathrm{~d} \boldsymbol{D}}=\left[\begin{array}{ll}
\frac{\partial L}{\partial \boldsymbol{x}} & \frac{\partial L}{\partial \boldsymbol{u}}
\end{array}\right]\left[\begin{array}{l}
\frac{\mathrm{d} \boldsymbol{x}}{\mathrm{d} \boldsymbol{D}} \\
\frac{\mathrm{d} \boldsymbol{u}}{\mathrm{d} \boldsymbol{D}}
\end{array}\right]
$$

As previously noted, the variables $\boldsymbol{u}$ and $\boldsymbol{x}$ represent the values over all time steps, and the inner products are over all space and time. The individual disciplinary sensitivities are given as the solution of the coupled system, which is obtained by differentiating the coupled analysis equations:
The first equation corresponds to equations for surface mesh point sensitivities with respect to the shape design variables, while the second equation rotates these sensitivities through the prescribed pitch angle, and adds the sensitivities with respect to the pitch control inputs, which are also used as design parameters. The third equation propagates the surface mesh sensitivities to the interior mesh points through the mesh deformation equations, while the fourth equation corresponds to the azimuthal rotation of these mesh sensitivities. The fifth equation generates the flow sensitivities based on the mesh sensitivities and the solution of the linearized flow problem. The flow sensitivities are then used to construct force sensitivities for the structural model using the FSI, which in turn generate structural sensitivities in the seventh equation. These are passed back to the CFD surface mesh in the last equation, to be reused at the next coupling iteration. As can be seen, each disciplinary solution procedure requires the inversion of the same Jacobian matrix as the corresponding analysis problem, which is done using the same solution technique.

Technically, the above system represents the forward sensitivity equations to be solved over all time steps. However, as mentioned previously, due to the dependence on at most the previous two time levels in all the individual disciplinary equations, the above matrix equation can be solved by forward substitution in time. The left-hand side matrix can rearranged into a lower triangular form, by reordering of the equations grouping together all disciplinary equations at a given time step, which are then solved simultaneously before advancing to the next time step, as described in references $[13,32]$. However, for convenience we retain the global temporal notation with the understanding that the above system can also be interpreted as the equations to be solved at a given time step, simply by adding the appropriate time level superscripts to the corresponding operators and variables.

The corresponding adjoint problem can be obtained by premultiplying the above matrix equation by the inverse of the large coupling matrix and substituting this into Eq. (27), transposing the entire system, and defining adjoint variables as solutions to the following coupled system:

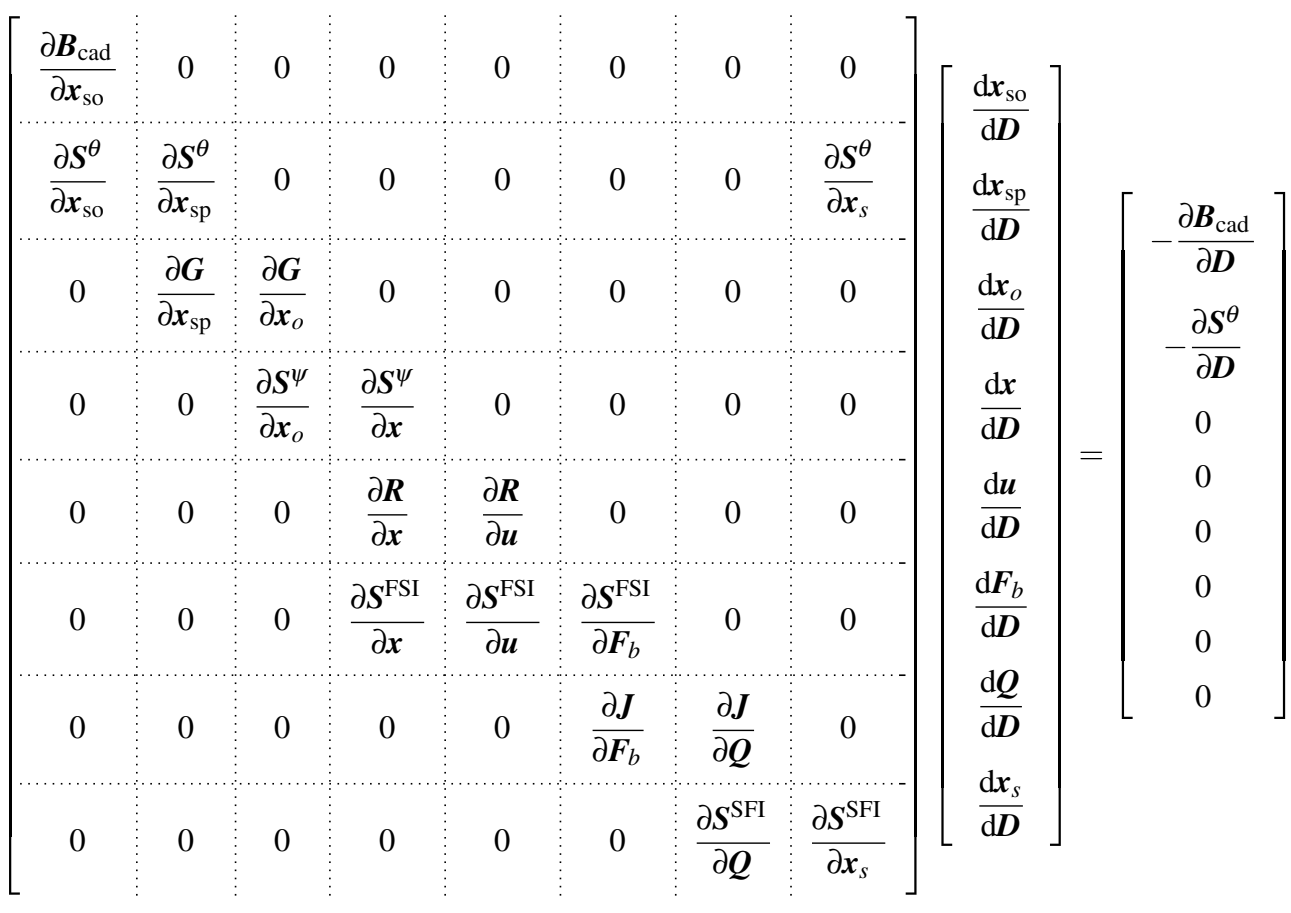




\begin{tabular}{|c|c|c|c|c|c|c|c|c|c|c|}
\hline${\frac{\partial \boldsymbol{B}_{\mathrm{cad}}}{\partial \boldsymbol{x}_{\mathrm{so}}}}^{T}$ & $\frac{\partial \boldsymbol{S}^{\theta^{T}}}{\partial \boldsymbol{x}_{\mathrm{so}}}$ & 0 & 0 & 0 & 0 & 0 & 0 & & & \\
\hline 0 & $\frac{\partial \boldsymbol{S}^{\theta^{T}}}{\partial \boldsymbol{x}_{\mathrm{sp}}}$ & ${\frac{\partial \boldsymbol{G}}{\partial \boldsymbol{x}_{\mathrm{sp}}}}^{T}$ & 0 & 0 & 0 & 0 & 0 & & & 0 \\
\hline 0 & 0 & $\frac{\partial \boldsymbol{G}^{T}}{\partial \boldsymbol{x}_{o}}$ & $\frac{\partial \boldsymbol{S}^{\psi^{T}}}{\partial \boldsymbol{x}_{o}}$ & 0 & 0 & 0 & 0 & $\Lambda_{x_{\mathrm{sp}}}$ & & $\begin{array}{l}0 \\
0\end{array}$ \\
\hline 0 & 0 & 0 & $\frac{\partial S^{\psi^{T}}}{\partial \boldsymbol{x}}$ & $\frac{\partial \boldsymbol{R}}{\partial x}^{T}$ & $\frac{\partial S^{\mathrm{FSI}}{ }^{T}}{\partial \boldsymbol{x}}$ & 0 & 0 & $\begin{array}{c}\Lambda_{x_{o}} \\
\Lambda_{x}\end{array}$ & & $\frac{\partial \boldsymbol{L}^{T}}{\partial \boldsymbol{x}}$ \\
\hline 0 & 0 & 0 & 0 & $\frac{\partial \boldsymbol{R}^{T}}{\partial \boldsymbol{u}}$ & $\frac{\partial \boldsymbol{S}^{\mathrm{FSI}}}{\partial \boldsymbol{u}}$ & 0 & 0 & $\begin{array}{c}\Lambda_{u} \\
\Lambda_{F_{b}}\end{array}$ & & $\frac{\partial \boldsymbol{L}^{T}}{\partial \boldsymbol{u}}$ \\
\hline 0 & 0 & 0 & 0 & 0 & $\frac{\partial \boldsymbol{S}^{\mathrm{FSI}}}{\partial \boldsymbol{F}_{b}}$ & ${\frac{\partial \boldsymbol{J}}{\partial \boldsymbol{F}_{b}}}^{T}$ & 0 & $\Lambda_{Q}$ & & $\begin{array}{l}0 \\
0\end{array}$ \\
\hline 0 & 0 & 0 & 0 & 0 & 0 & $\frac{\partial \boldsymbol{J}}{\partial \boldsymbol{Q}}^{T}$ & $\frac{\partial \boldsymbol{S}^{\mathrm{SFI}}}{\partial \boldsymbol{Q}}$ & & & 0 \\
\hline 0 & $\frac{\partial \boldsymbol{S}^{\theta^{T}}}{\partial \boldsymbol{x}_{s}}$ & 0 & 0 & 0 & 0 & 0 & $\frac{\partial \boldsymbol{S}^{\mathrm{SFI}}}{\partial \boldsymbol{x}_{S}}$ & & & \\
\hline
\end{tabular}

Once again, the solution of the various disciplinary adjoint equations requires the inversion of the corresponding disciplinary Jacobians (transposed in this case), which can be accomplished using the same iterative solvers as for the analysis and forward sensitivity problems, but proceeding in the reverse order. By analogy with the forward sensitivity problem, this coupled adjoint system over all time steps can be written in upper triangular form and solved by back substitution, which corresponds to a backward integration in time $[13,32]$. The specific coupled system to be solved at each time step can be recovered by specifying the time-level superscripts on all operators and variables.

Once the solution of the coupled disciplinary adjoint problems has been obtained, these can be used to compute the objective sensitivities as

$$
\frac{\mathrm{d} L}{\mathrm{~d} \boldsymbol{D}}=\left[\begin{array}{llllllll}
-\frac{\partial \boldsymbol{B}_{\mathrm{cad}}}{\partial \boldsymbol{D}} & -\frac{\partial \boldsymbol{S}^{\theta} \boldsymbol{D}}{\partial \boldsymbol{D}} & 0 & 0 & 0 & 0 & 0 & 0
\end{array}\right]\left[\begin{array}{c}
\Lambda_{x_{\mathrm{so}}} \\
\Lambda_{x_{\mathrm{sp}}} \\
\Lambda_{x_{o}} \\
\Lambda_{x} \\
\Lambda_{u} \\
\Lambda_{F_{b}} \\
\Lambda_{Q} \\
\Lambda_{x_{s}}
\end{array}\right]
$$

Figure $\underline{3}$ illustrates the flow of information between fluid and structural disciplines for the analysis, tangent, and adjoint solvers. In the analysis problem, the CFD solver sends a force vector to the structural solver and receives a surface displacement vector; whereas in the tangent problem, these are replaced with a force sensitivity and displacement sensitivity vector, respectively. For the adjoint problem, the information proceeds in reverse order, with the CFD solver receiving a force adjoint vector from the structural model and sending a displacement adjoint to the structural model. In all cases, these vectors have the same dimensions as those used in the analysis problem and make use of the same data structures for interdisciplinary coupling. The unsteady adjoint requires a solution state from each of the time steps, which is easily read from a hard disk saved during the forward analysis step. This avoids additional memory requirements.
The forward and adjoint sensitivities for the coupled aeroelastic problem are verified using the complex step method [33]. As in the case of finite differencing, the complex step-based differentiation also requires a step size. However, unlike finite differencing, the complex step method is insensitive to small step sizes because no differencing is required. In theory, it is possible to verify forward and adjoint-based gradients using the complex step method to machine precision. With this in mind, a complex version of the complete coupled aerostructural analysis code has been constructed through scripting of the original source code to redefine variables from real to complex types and to overload a small number of functions for use with complex variables.

\section{Results}

\section{A. Time-Dependent Analysis Problem}

The chosen test case is a four-bladed HART-II rotor in a forwardflight condition. The flight conditions correspond to a wind-tunnel

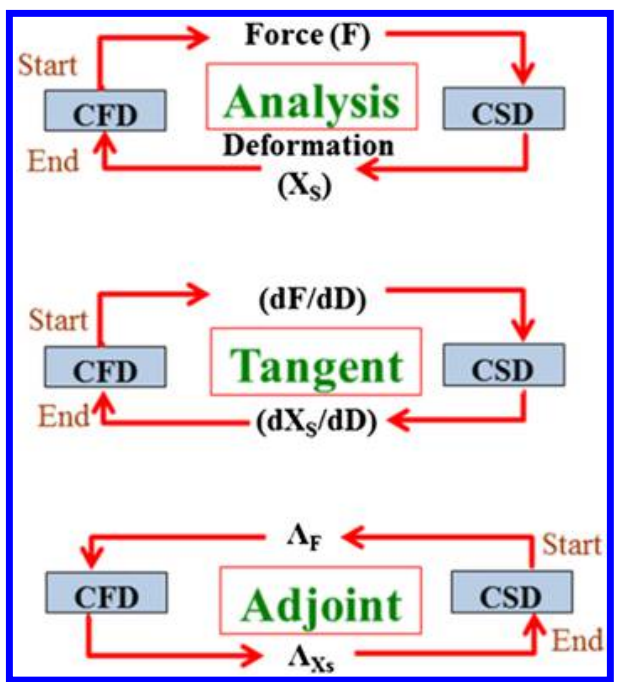

Fig. 3 Corresponding flow of information for analysis, tangent, and adjoint solution processes. 


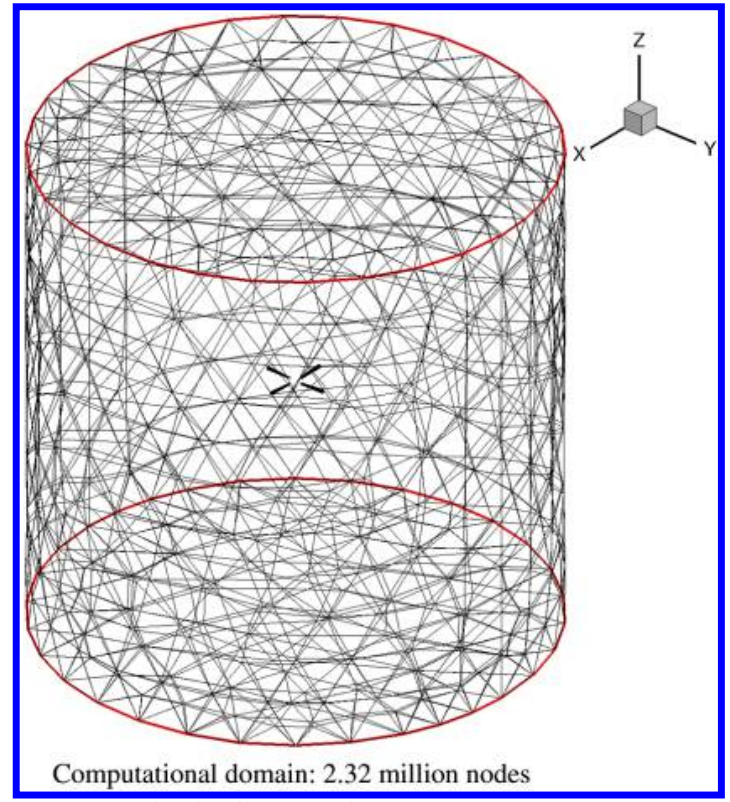

Fig. 4 Computational polyhedra mesh.

test [28] with a freestream Mach number of $M_{\infty}=0.095$, a rotor tip Mach number of $M_{\text {tip }}=0.638$, and a shaft tilt angle toward a freestream of $\alpha_{\text {shaft }}=5.4 \mathrm{deg}$. The corresponding rotor rotational speed is $\Omega=1041 \mathrm{rpm}$. The blade pitch angle actuation is prescribed as $\theta=\theta_{0}+\theta_{1 s} \sin (\psi)+\theta_{1 c} \cos (\psi)$, with $\theta_{0}=5.0 \mathrm{deg}$, $\theta_{1 s}=-1.1 \mathrm{deg}$, and $\theta_{1 c}=2.0 \mathrm{deg}$, where $\psi$ corresponds to the azimuthal angle of rotation about the hub axis. The rotor is impulsively started from rest, in an initially quiescent flowfield, and rotated with the mesh as a solid body for a fixed number of revolutions. This problem is solved both for a rigid-blade model (using no structural model) as well as for a flexible-blade model (using the beam structural model). For the latter, the flow is solved in a tight coupling mode with the beam solver as described previously.

The baseline simulation (coarse mesh) makes use of a mixed element mesh made up of prisms, pyramids, and tetrahedra consisting of approximately 2.32 million grid points and is shown in Fig. 4a. The simulations are run for five rotor revolutions using a 2 deg time step, $i$. e., for 900 time steps starting from freestream initialization. For the rigid-blade simulation, the time-dependent mesh motion is determined by first pitching the blade about the blade axis followed by solving the mesh deformation equations and then rotating the entire mesh as a solid body at each time step. The unsteady Reynoldsaveraged Navier-Stokes equations are solved at each time step in ALE form, using the Spalart-Allmaras turbulence model.
The coupled CFD/CSD simulation is run in a similar manner. However, the flow solution (CFD) is coupled with the beam solver (CSD) at every time step by appropriately exchanging 1) airloads information from the flow domain to the beam; and 2) blade deformation information from the beam to the flow domain, through the fluid-structure interface (i.e., blade surface). In this coupled simulation, the mesh is moved according to the deformations dictated by the new flexed blade coordinates determined from the structural beam code after the combined kinematics of pitch actuation followed by solid body rotation of the entire mesh have been performed. This coupled fluid-structure interaction problem needs to be iterated until satisfactory convergence (up to more than two orders of magnitude drop) is achieved on flow, structure, and mesh deformation problems within each time step. The flow and mesh deformation problems are converged up to more than two orders of magnitude of residual values. However, the structure problem is converged up to machine precision residual values which is possible due to the use of direct matrix inversion.

The simulations were performed on the Yellowstone supercomputer at the NCAR-Wyoming Supercomputing Center, with the analysis problem running in parallel on 1024 cores. Each time step used six coupling iterations and each coupling iteration used 10 nonlinear flow iterations, with each nonlinear iteration consisting of a three-level line-implicit multigrid cycle. The typical simulation at this level of resolution required approximately $40 \mathrm{~min}$ of wall-clocktime per rotor revolution.

Figures 5 and 6 summarize the overall convergence of the rigid and aeroelastic coupling analysis formulations. Figure 5 a shows the typical flow and turbulence residual convergence within a single time step for the rigid rotor case (no structural model), whereas Fig. $5 \mathrm{~b}$ depicts the convergence of the flow and turbulence residuals at the same time step for the coupled aeroelastic case. In this case, the jumps in residual values at the start of new coupling iterations are clearly visible, although these jumps become smaller as the coupling procedure converges. The overall residual histories closely follow those of the rigid rotor case after the first few coupling iterations. Figure 6 a depicts the convergence of the mesh deformation residual for the same time step, also showing jumps in the residual at the start of each new coupling iteration. Solution of the mesh deformation equations terminates when the residuals reach a prescribed tolerance of $10^{-6}$ (lowest residual shown in the figure is greater than $10^{-6}$ ), and hence the variable number of iterations per coupling cycle. Most notable is the fact that the initial mesh deformation residual decreases at each new coupling iteration, providing a measure of the convergence of the entire coupling procedure. The corresponding residual drop for the beam equations, which is solved by direct inversion, is observed to be of 15 orders of magnitude, as shown in Fig. $6 \mathrm{~b}$. Figure $6 \mathrm{~b}$ illustrates the convergence of the coupled beam/ FSI residual [i.e., Eqs. (24) and (25)], showing rapid convergence to

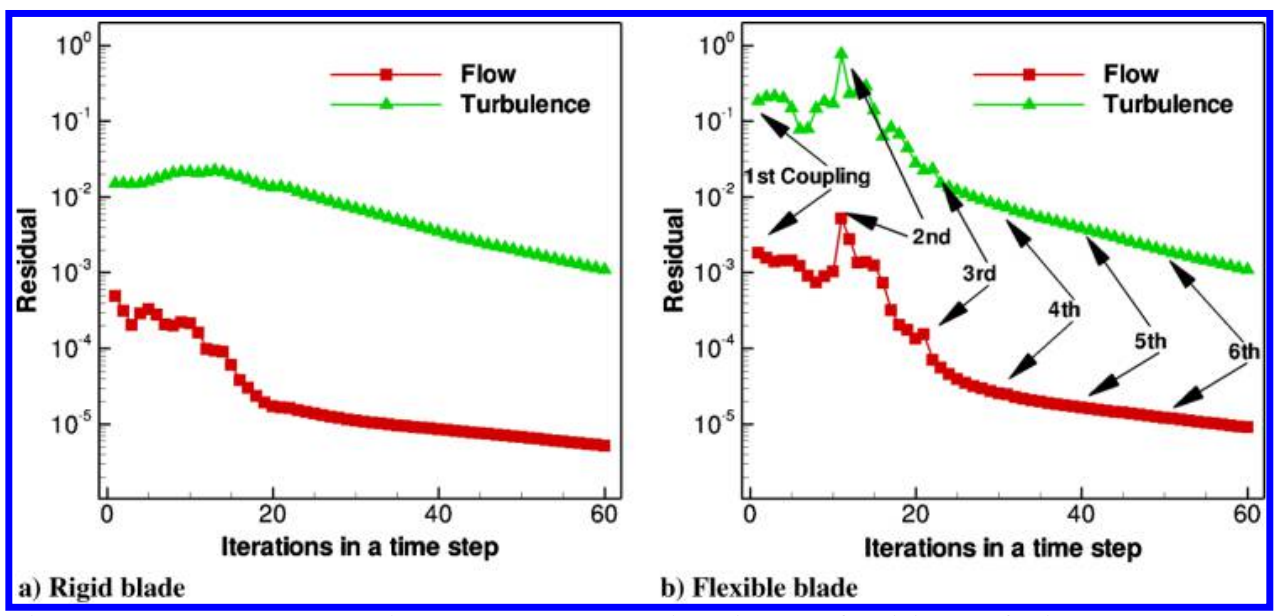

Fig. 5 Flow and turbulence residual convergence at a given time step for rigid- and flexible-blade analyses. 


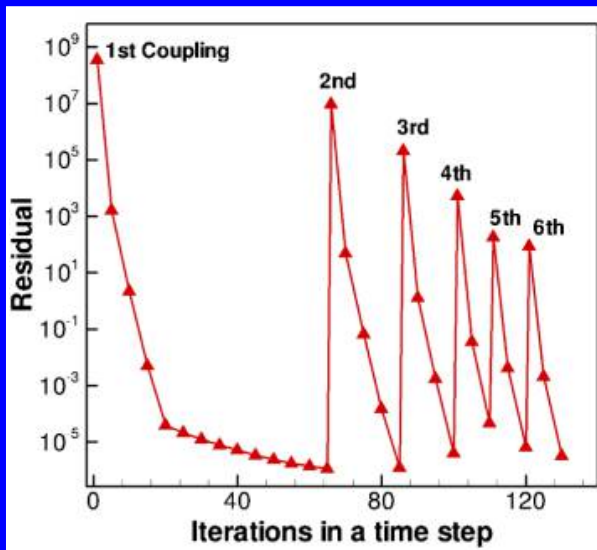

a) Mesh convergence per time step for coupled analysis

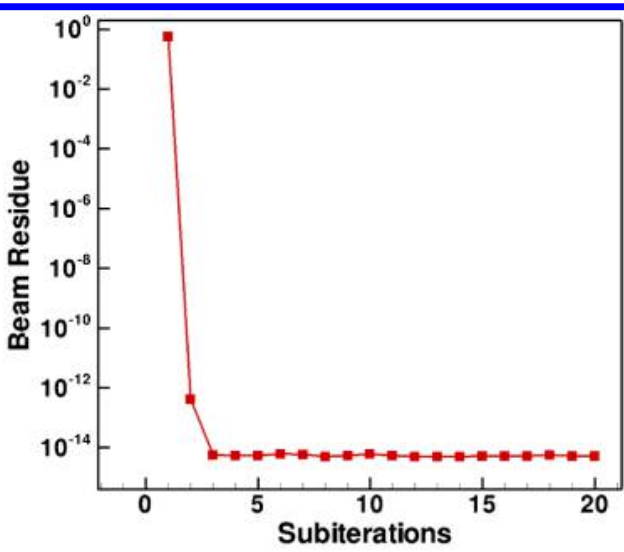

b) Beam and FSI convergence per coupling iteration

Fig. 6 Residual convergence of mesh and beam (overall FSI) in one coupling iteration.

machine zero in a small number of iterations within a single CFD/ CSD coupling iteration.

The effect of the CFD/CSD aeroelastic coupling is clearly demonstrated in Fig. 7a, which compares the deformed blade shape and its corresponding $C_{p}$ surface contours from the coupled simulation with that from the rigid-blade simulation. From the figure, it is noted that all four blades show different deformation characteristics due to corresponding different aerodynamic environments they experience in forward flight. The blade attains the largest flap displacement at azimuth $(\psi=180 \mathrm{deg})$ and the smallest flap displacement at azimuth $(\psi=$ $0 \mathrm{deg}$ ). For both the flexible and rigid blades, the pressure contours demonstrate that the advancing side blades experience higher loading (larger pressure gradients near the rotor tips) than the retreating side blades. The flexible-blade tip vertical displacement time history shown in Fig. $7 \mathrm{~b}$ demonstrates 1 per cycle behavior of blade flapping, as expected.

Figure 8 summarizes the performance comparison between flexible and rigid blades over five rotor revolutions. Besides showing consistent $4 / \mathrm{rev}$ harmonic content in the airloads, the figures also show that the rigid blades overpredict thrust and power compared to flexible blades. This suggests that performance predictions might be erroneous if the aeroelastic nature of rotor blade is not taken into account. Figure 9 further compares the rotor longitudinal $C_{M_{Y}}$ and lateral $C_{M_{X}}$ moments of the flexible rotor with the rigid rotor. It should be noted that neither the rigid nor the flexible rotor are in a moment trimmed state (zero mean moment) at the prescribed pitch parameters.
B. Fully Coupled Unsteady Aeroelastic Adjoint Sensitivity Verification

\section{Geometry Parameterization}

To obtain sensitivities with respect to a set of shape parameters that are well suited for design optimization purposes, a baseline blade is constructed by stacking 11 airfoil sections along the span. Each airfoil contains 10 Hicks-Henne bump functions (five on the upper surface, and five on the lower surface) that can be used to modify the airfoil shape. Additionally, the twist values of the blade at the root and tip airfoil sections are also used as design variables, resulting in a total of 112 design variables. Figure 10a provides an illustration of the baseline blade design setup. A high-density structured mesh is generated about this blade geometry, which is then rotated and translated to match each individual blade in the CFD mesh, as shown in Fig. 10b. Interpolation patterns between each unstructured mesh surface point and the baseline structured mesh are determined in a preprocessing phase. These interpolation patterns are then used to interpolate shape changes from the baseline blade to all four blades in the CFD mesh (as determined by changes in the design variables) and to transfer sensitivities from the surface CFD mesh points to the design variables using the chain rule of differentiation.

In addition to the aforementioned design variables, the inclusion of control parameters for pitching in forward flight introduces a new set of three design parameters, i.e., $\boldsymbol{D}=\left[\theta_{0}, \theta_{c_{1}}, \theta_{s_{1}}\right]$. Design variable $\theta_{0}$ is known as rotor collective pitch angle, and $\theta_{c_{1}}$ and $\theta_{s_{1}}$ are the rotor cyclic pitch angles. This results in a total of 115 design variables. These sensitivities, in addition to the aforementioned geometric sensitivities, are transferred onto the CFD surface mesh points from

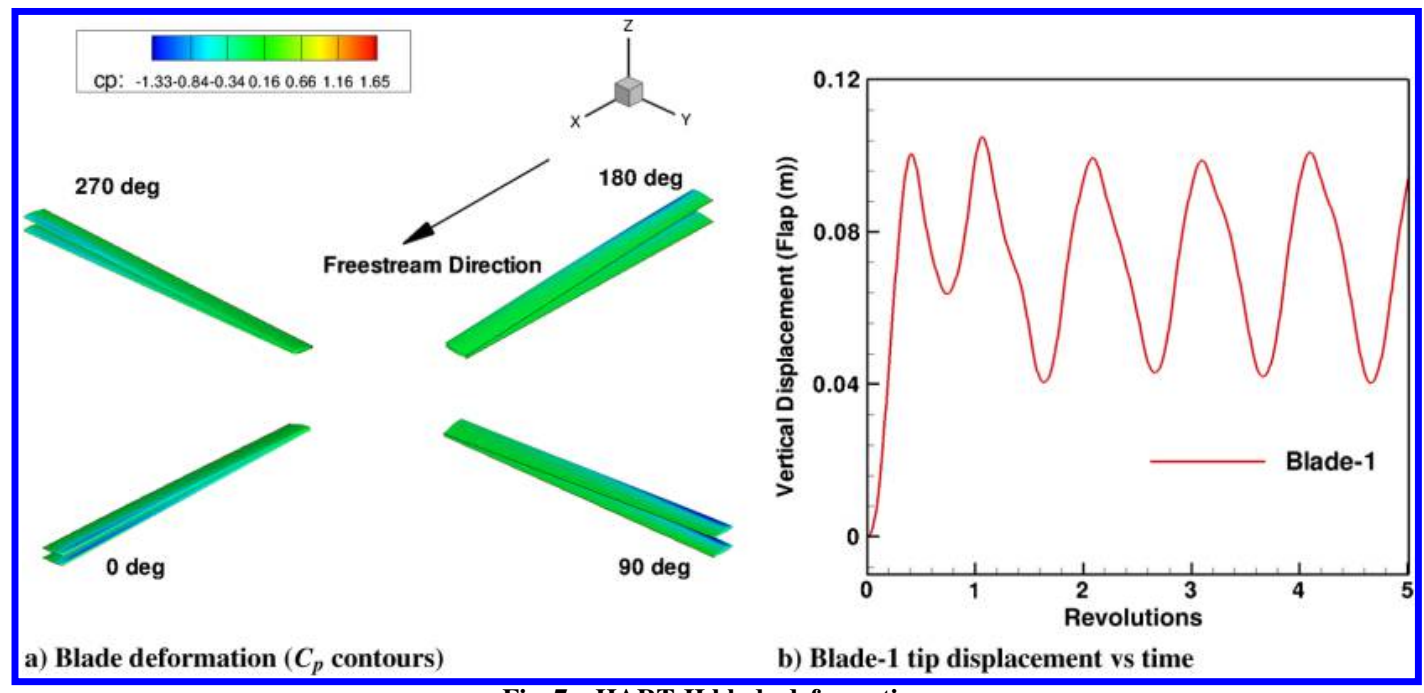

Fig. 7 HART-II blade deformation. 


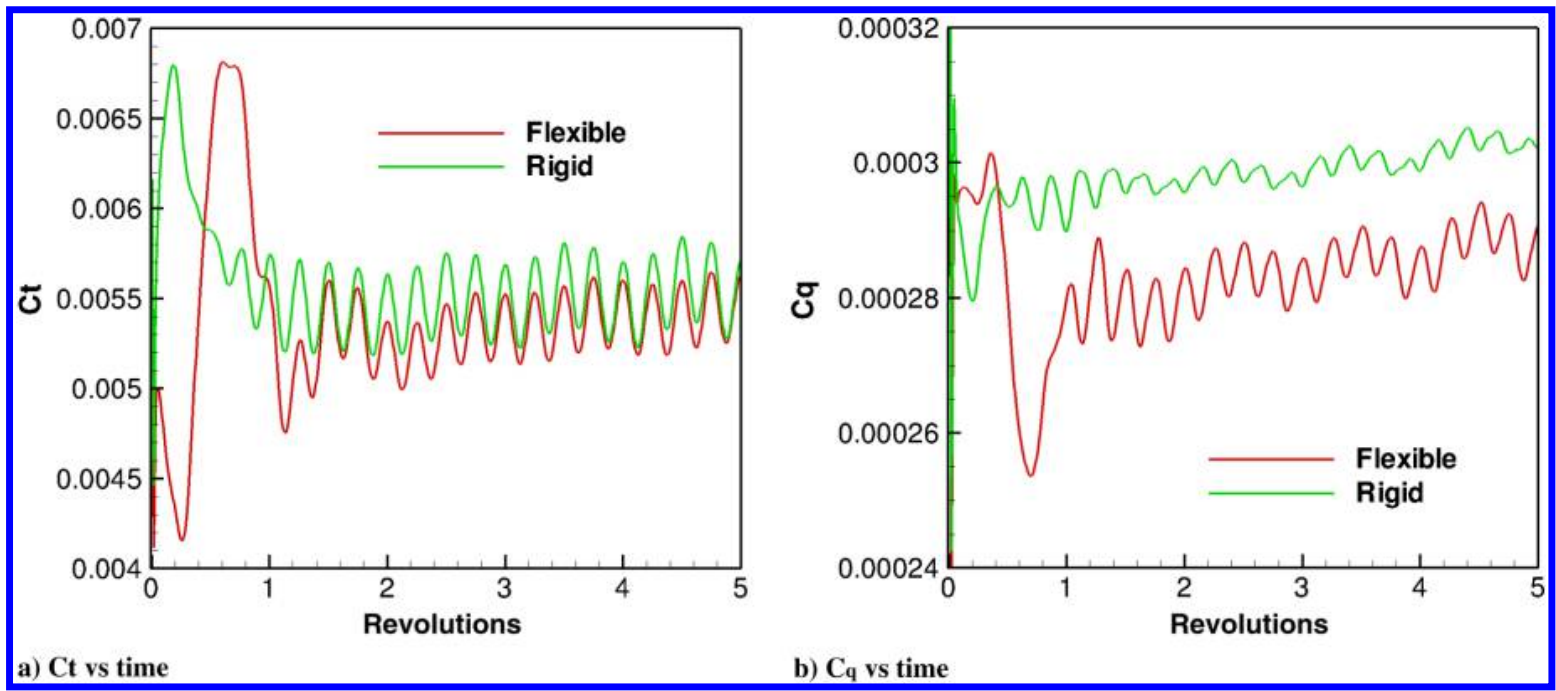

Fig. 8 Performance comparison of flexible blade with rigid blade.

the master blade shape through the interpolation method described earlier.

\section{Unsteady Objective Function Formulation}

A time-integrated objective function based on the time variation of the thrust $C_{T}$, torque $C_{Q}$, and moment coefficients $\left(C_{M_{X}}\right.$ and $\left.C_{M_{Y}}\right)$ is used for this test case. The goal of the optimization is to reduce the timeintegrated torque coefficient while constraining the time-integrated thrust coefficient to the baseline rotor performance, as well as constraining the moment coefficients along the roll $C_{M_{X}}$ and pitch $C_{M_{Y}}$ axes of the rotor to a trimmed value, i.e., zero average moment values. The objective function is based on the summation of the differences
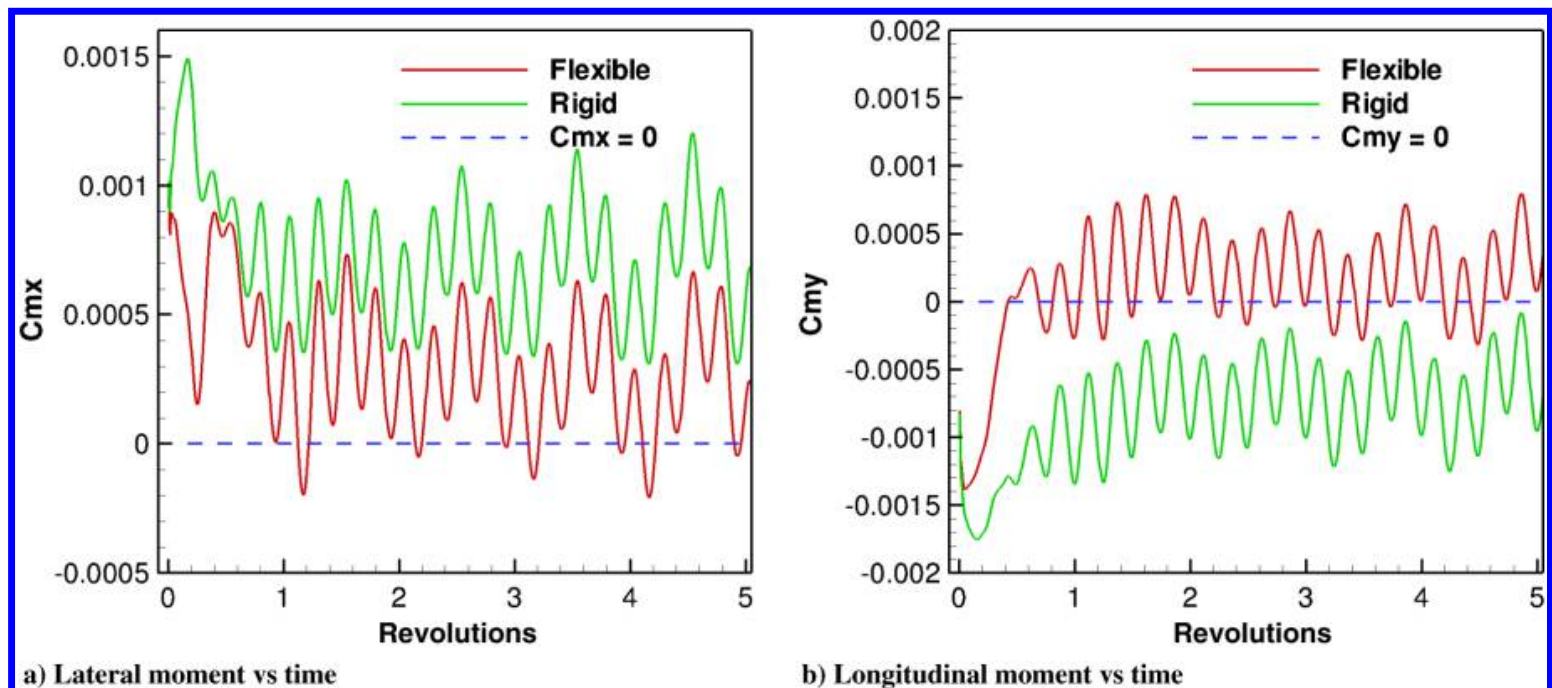

b) Longitudinal moment vs time

Fig. 9 Rotor moments comparison of flexible blade with rigid blade.
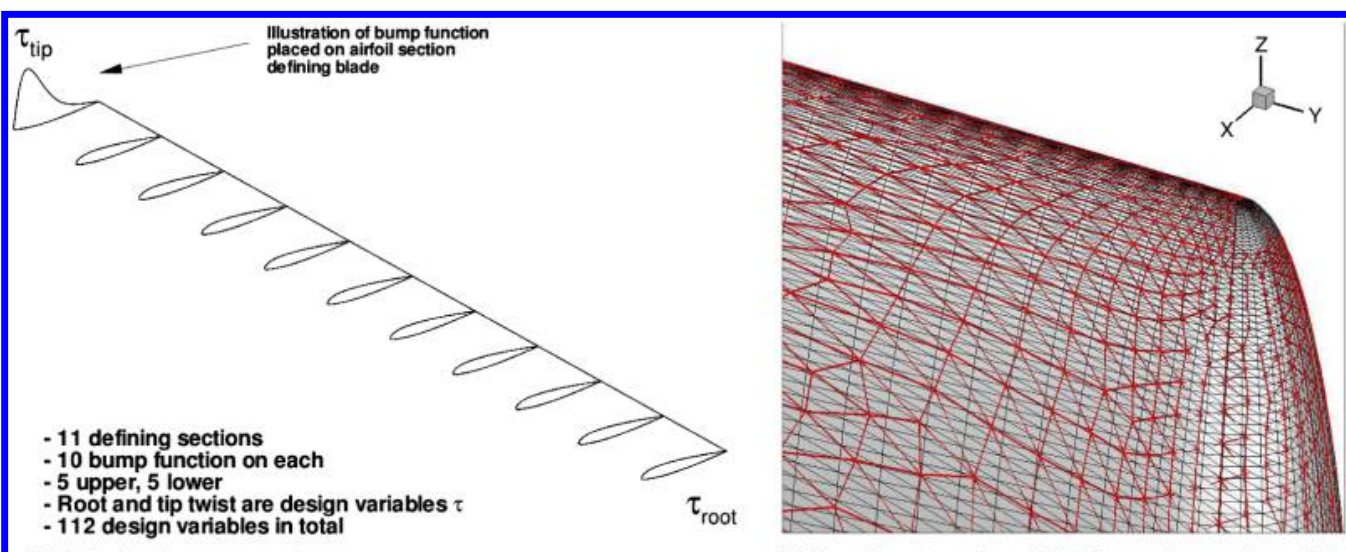

a) Blade design parameters

b) Baseline structured blade mesh overlap with CFD mesh

Fig. 10 Illustration of a) baseline blade with design parameters and b) overlap in tip region between baseline blade structured mesh and CFD surface unstructured mesh. 
between a target and a computed objective value at each time step $n$. Mathematically, the global objective function is defined as

$$
\begin{gathered}
L^{g}=L_{\text {Shape }}+L_{\text {Trim }} \\
L_{\text {Shape }}=\omega_{1} \frac{1}{T} \sum_{n=1}^{n=N} \Delta t\left[\delta C_{Q}^{n}\right]^{2} \\
L_{\text {Trim }}=\omega_{2} \frac{1}{T} \sum_{n=1}^{n=N} \Delta t\left[\delta C_{T}^{n}\right]^{2}+\omega_{3}\left[\delta \bar{C}_{M_{X}}\right]^{2}+\omega_{4}\left[\delta \bar{C}_{M_{Y}}\right]^{2} \\
\delta C_{Q}^{n}=\left(C_{Q}^{n}-C_{Q \text { target }}^{n}\right) \\
\delta C_{T}^{n}=\left(C_{T}^{n}-\bar{C}_{T \text { target }}\right) \\
\delta \bar{C}_{M_{X}}=\frac{1}{T} \sum_{n=1}^{n=N} \Delta t\left(C_{M_{X}}^{n}-C_{M_{X} \text { target }}^{n}\right) \\
\delta \bar{C}_{M_{Y}}=\frac{1}{T} \sum_{n=1}^{n=N} \Delta t\left(C_{M_{Y}}^{n}-C_{M_{Y} \text { target }}^{n}\right)
\end{gathered}
$$

where the mean target thrust coefficient value is specified to the HARTII baseline mean trim value of $\bar{C}_{T \text { target }}=0.0044$, and the target torque and moment values are set to zero. The weights $\left(\omega_{i}, i=[1,2,3,4]\right)$ are included to equalize the difference in orders of magnitude between the thrust, torque, and moment coefficients. Use of pitch control parameters $\left(\left[\theta_{0}, \theta_{c_{i}}, \theta_{s_{i}}\right]\right)$ as design variables and use of moment penalty terms [34] ensure that the optimized rotor shape with optimized control parameters tend toward a final trimmed state when the rotor design cycles converge. Ideally, this penalty approach will be expected to be computationally more efficient than the use of a hard constraint formulation, since this latter approach will require the computation of multiple adjoint problems at each design cycle, as opposed to the single adjoint required in the current formulation. However, since the trim constraint may not be satisfied exactly in penalty approach, additional computational costs may be incurred in trimming the rotor (using a trim optimization step) before shape design is carried out. Given only a few number of iterations required for trim optimization, the overall penalty approach can still be more efficient. Although, some references [35] claimed to have achieved hard constraint design optimization solving multiple adjoints with only an incremental cost, it remains unclear if this additional cost using their suggested approach would be any less than that required in trim step.

\section{Unsteady Adjoint Sensitivity Verification}

The fully coupled CFD/CSD adjoint formulation was verified by comparing its sensitivities with those obtained from the tangent as well as the complex step method. The coupled adjoint formulation was verified for perturbations of the collective pitch parameter $\theta_{0}$ and one shape design parameter (blade twist), for both uncoupled (rigid blade) as well as coupled (flexible blade) simulations. A complex perturbation of size $1 \times 10^{-100}$ is introduced on the collective pitch at the beginning of the analysis run. The design sensitivities of the functional $\left(\partial L^{g} / \partial D\right)$ obtained from complex, tangent, and adjoint formulations are compared after every time instance for up to 180 time steps (one full rotor revolution) for both the design parameters, as shown in Tables 1 and 2 . Both the rigid and the fully coupled aeroelastic problems āre converged to machine zero at each time step

\begin{tabular}{|c|c|c|c|}
\hline$\underline{n}$ & Method & Uncoupled (rigid) & Coupled (aeroelastic) \\
\hline \multirow[t]{3}{*}{1} & $\mathrm{C}$ & 8. & 7.5. \\
\hline & & & \\
\hline & Adjoint & 8.135662924562 884E-005 & 7.56981714367 2761E-005 \\
\hline \multirow[t]{3}{*}{2} & & & 6.040142774935 852E-005 \\
\hline & & & 05 \\
\hline & Adj & 05 & 005 \\
\hline \multirow[t]{3}{*}{3} & Complex & 277E-005 & -4.95990987078 6381E-006 \\
\hline & & & -4 . \\
\hline & Adj & 05 & -006 \\
\hline \multirow[t]{3}{*}{4} & $\mathrm{Co}$ & 005 & 005 \\
\hline & & -3 & \\
\hline & Adj & -3.27 & -005 \\
\hline \multirow[t]{3}{*}{5} & Complex & -7.4662642 & -1.142069116982 308E-004 \\
\hline & & -7.4 & -004 \\
\hline & Adjoint & -7.466264 & -1.142069116982 432E-004 \\
\hline \multirow[t]{3}{*}{180} & Complex & -2.60622548 & -5.1761894274 \\
\hline & & -2.6 & \\
\hline & & $-2.606225487528119 \mathrm{E}-003$ & 2743 4507E-003 \\
\hline
\end{tabular}
in order to avoid contaminating the sensitivity values with errors due to incomplete convergence. The rigid sensitivity verification serves

\begin{tabular}{|c|c|c|c|}
\hline$n$ & Method & Uncoupled (rigid) & Coupled \\
\hline \multirow[t]{3}{*}{1} & lex & 6 & 1. \\
\hline & Tan & 76E-006 & 76191 9181E-006 \\
\hline & Adjoint & 1.1637068616115 53E-006 & 376191 9045E-006 \\
\hline \multirow[t]{3}{*}{2} & Complex & 301 53E-006 & 8.5786 \\
\hline & Tangent & 1.0761441 & 8.578611087 \\
\hline & Adjoint & $1.076144190530128 \mathrm{E}-006$ & $8.578611087035991 \mathrm{E}-007$ \\
\hline \multirow[t]{3}{*}{3} & Complex & 4.142066931 & 1.17278 \\
\hline & Tan & 007 & E-007 \\
\hline & Adjoint & 4.142 & -007 \\
\hline \multirow[t]{3}{*}{4} & Complex & $-1.846822818931199 \mathrm{E}-007$ & $5110603 \mathrm{E}-007$ \\
\hline & Tan & E-007 & E-007 \\
\hline & Adjoint & $-1.846822818929633 \mathrm{E}-007$ & -5.751866415 041355E-007 \\
\hline \multirow[t]{3}{*}{5} & Complex & -7.0780547287 71792E-007 & -1.2194927006 26512E-006 \\
\hline & & -007 & \\
\hline & Adjoint & 141E-007 & BE-006 \\
\hline \multirow[t]{3}{*}{180} & Complex & $-3.170858753236345 \mathrm{E}-005$ & -6.584007142079 \\
\hline & & $-3.170858753236326 \mathrm{E}-005$ & $-6.584007142065199 \mathrm{E}-005$ \\
\hline & Adjoint & -3.17085875323 3577E-005 & -6.5840071420 75842E-005 \\
\hline
\end{tabular}
as an additional check of the forward sensitivity formulation for forward-flight conditions when the structural code is switched off. As can be seen from the table, the sensitivities agree to at least 11
Table 1 Adjoint sensitivity verification for forward flight $\mathrm{d} L / \mathrm{d} \Theta_{0}$

Table 2 Adjoint sensitivity verification for forward flight $\mathrm{d} L / \mathrm{d} \theta_{\text {twist }}$

Bold text helps in displaying the precision of comparison between the three differen sensitivity values (complex vs tangent vs adjoint)

significant digits for both the rigid and flexible aeroelastic rotor cases. More important, the agreement does not degrade with an additional number of time steps, and it remains close to machine precision over a full rotor revolution, as performed in this case.

\section{Performance Optimization of Helicopter Rotor in Forward Flight}

The optimization tool thus verified is applied to the flexible HARTII rotor using the same time-dependent test case as described in Sec. V.A. The simulation is run for two full rotor revolutions, starting impulsively from rest in quiescent flow. The objective consists of the time-integrated torque with a thrust and moment penalty, as described previously. However, the objective to be minimized is only integrated over the last rotor revolution, in order to avoid the optimization process from focusing on startup transients. Although a larger number of rotor revolutions will be required to ensure periodic solution and be more appropriate for trim optimization, in the view of demonstrating adjoint capability, the number of rotor revolutions is restricted to only two in the view of demonstrating adjoint optimization capability. Moreover, for the flexible blades, the test problem aims at addressing the unsteady adjoint solution during the transient state in the first two revolutions, which differs significantly from the rigid-blade solutions. The optimization problem is solved on a relatively coarse grid (2.3 million points) using a time step size of $2.0 \mathrm{deg}$. Figure 11 


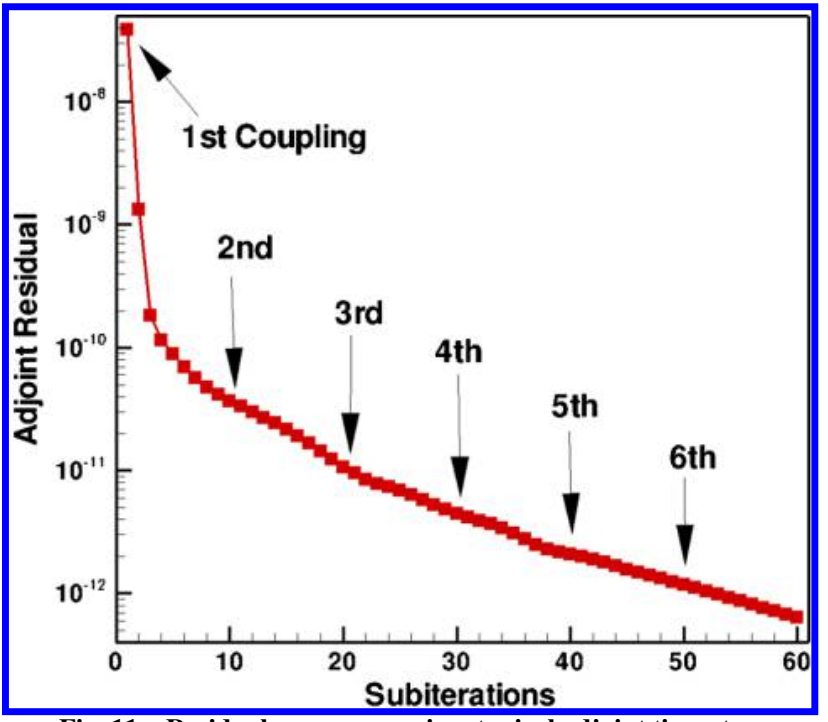

Fig. 11 Residual convergence in a typical adjoint time step.

shows the residual convergence for a typical unsteady adjoint time step for $\Delta t=2 \mathrm{deg}$. The figure shows the residual drops by five orders of magnitude over six coupling cycles.

The optimization procedure used is the Limited memory Broyden-Fletcher-Goldfarb-Shanno Bound constraint optimization bounded reduced Hessian algorithm [36]. Each request by the optimization driver for a function and gradient value results in a single forward time integration of the analysis solver and a single backward integration in time of the adjoint solver. A bound of $\pm 5 \%$ chord for each defining airfoil section is set on the Hicks-Henne [37] bump functions, a bound of $\pm 1.0 \mathrm{deg}$ of twist is set on the root and tip twist definitions, and a bound of $\pm 5.0 \mathrm{deg}$ of pitch angle is set on all the pitch parameters (collective and cyclic). The optimizations are performed on the Yellowstone supercomputer at the National Center for Atmospheric Research (NCAR)-Wyoming Supercomputing Center with the simulations (analysis/adjoint) running in parallel on 1024 cores. Each time step in the analysis problem employs six coupling cycles. Each coupling cycle uses 10 nonlinear iterations. A typical coupled functional gradient (analysis/adjoint) computation step requires approximately $70 \mathrm{~min}$ when run on 1024 cores.

The performance optimization consists of three main stages: 1) trim, 2) shape/performance optimization and, 3) retrim. The "trim" step involves trimming the rotor to a target windtunnel rotor thrust value of $C_{T}=0.0044$ and zero longitudinal and lateral moments $\left(C_{M_{Y}}, C_{M_{X}}=0\right)$. The objective function used in this step consists only of the thrust and moment terms, or simply $L_{\text {Trim }}$ in Eq. (29); and the objective minimization is performed using only the three pitch parameters, namely, collective and cyclic as design variables.

In the second stage, blade shape optimization is performed by including the performance objective, i.e., the $C_{Q}$ term into the timedependent objective function to be minimized. Appropriate weights $\left(\omega_{i}, i=1,2,3,4\right)$ are used to maintain the rotor trim state through a penalty function while the blade shape is optimized to obtain minimum rotor power. In total, 115 design parameters are used in this stage, including 112 blade shape parameters and three pitch control parameters. However, even after optimization convergence in stage two, the exact trim state is not maintained. This is because the trim objective components in the objective function are used only as weak constraint terms, i.e., as penalty terms and not as hard constraints. Therefore, the last stage involves trimming the rotor back to the target thrust and moment values, once again using only the three control

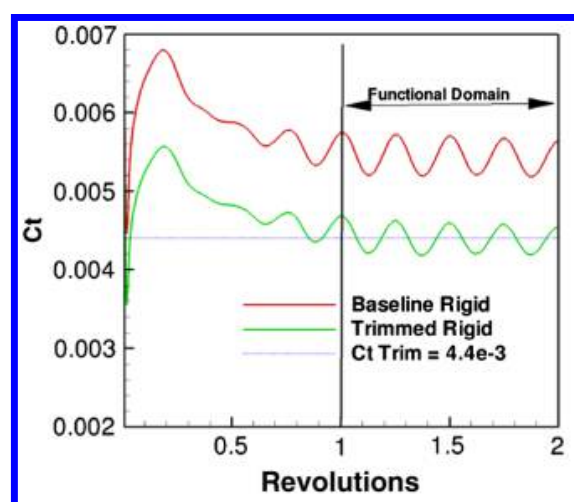

a) Thrust trim

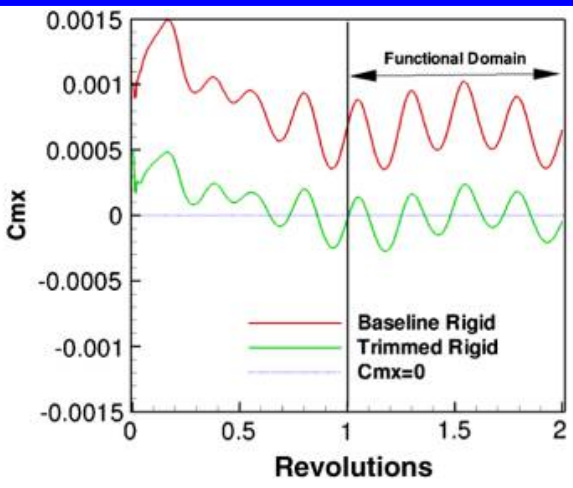

b) Lateral moment trim

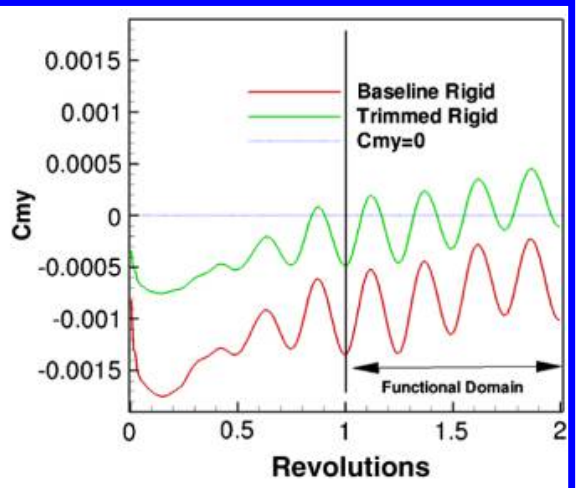

c) Longitudinal moment trim

Fig. 12 HART-II rigid-blade trim.

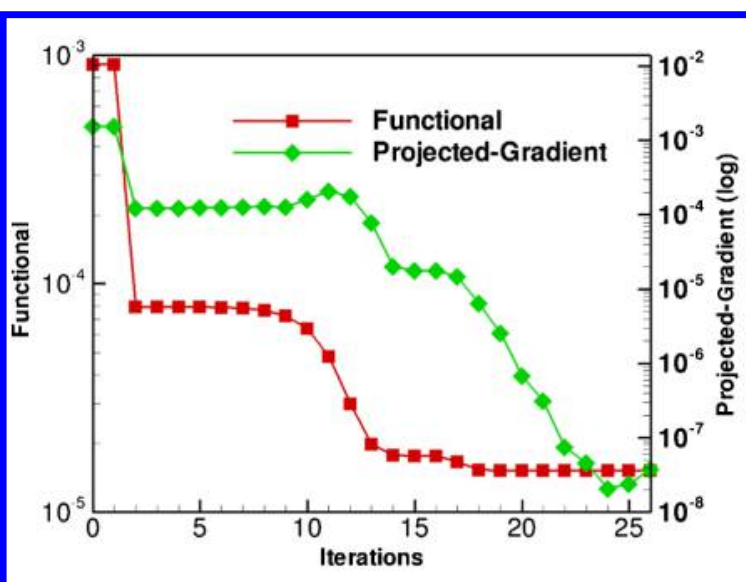

a) Trim optimization convergence

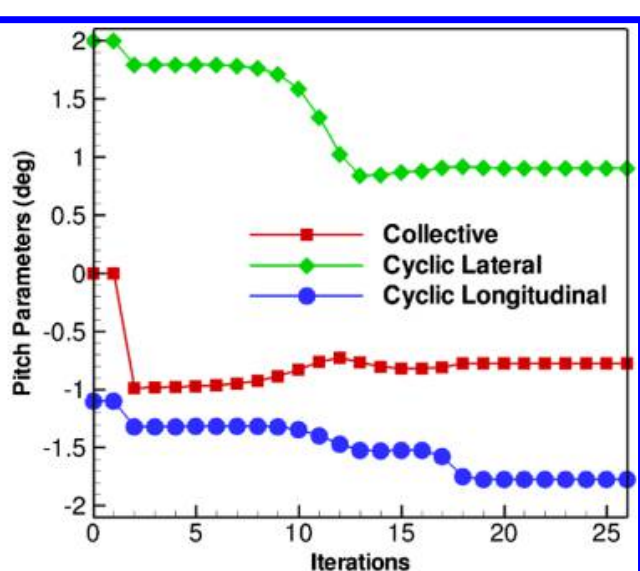

b) Pitch parameters convergence 


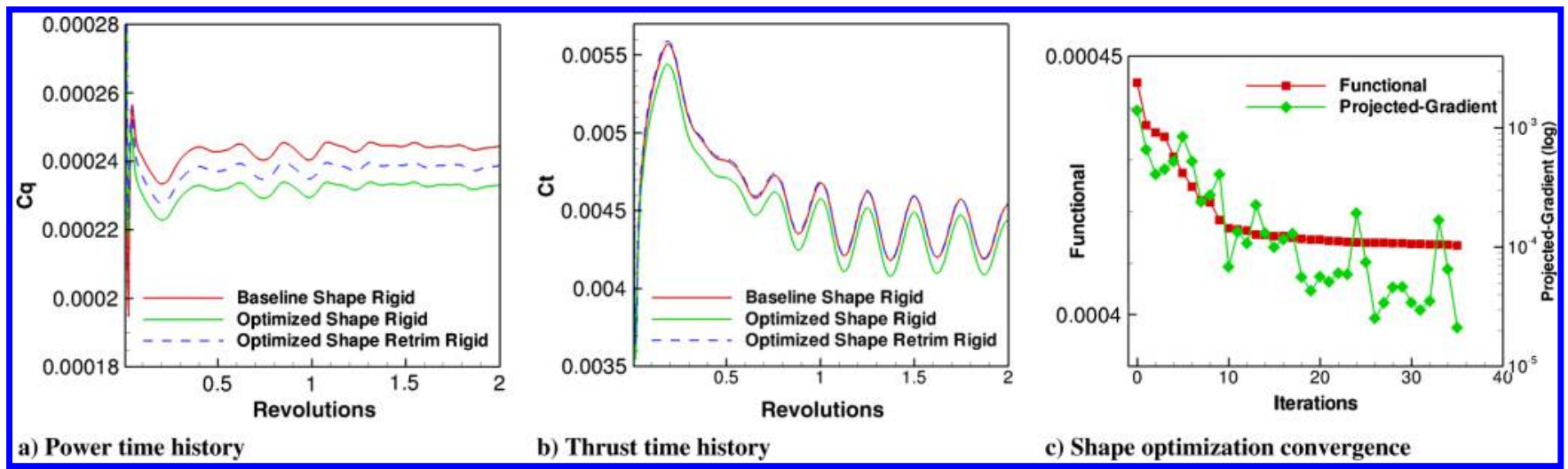

Fig. 14 HART-II rigid-blade shape optimization and retrim.

pitch parameters as design inputs. This stage is otherwise referred to as the "retrim" stage in this paper.

The optimization results for the rigid-blade case are presented first, followed by the flexible-blade optimization results. Figure 12 demonstrates that the rigid HART-II rotor was successfully trimmed to the target rotor thrust $\left(C_{T}=0.0044\right)$ and moment values $\left(C_{M_{Y}}\right.$, $C_{M_{Y}}=0$ ). Figure 13 a shows that the trim objective gradient drops by more than five orders of magnitude, whereas the objective functional achieves a minimum over 26 design iterations. The gradients shown

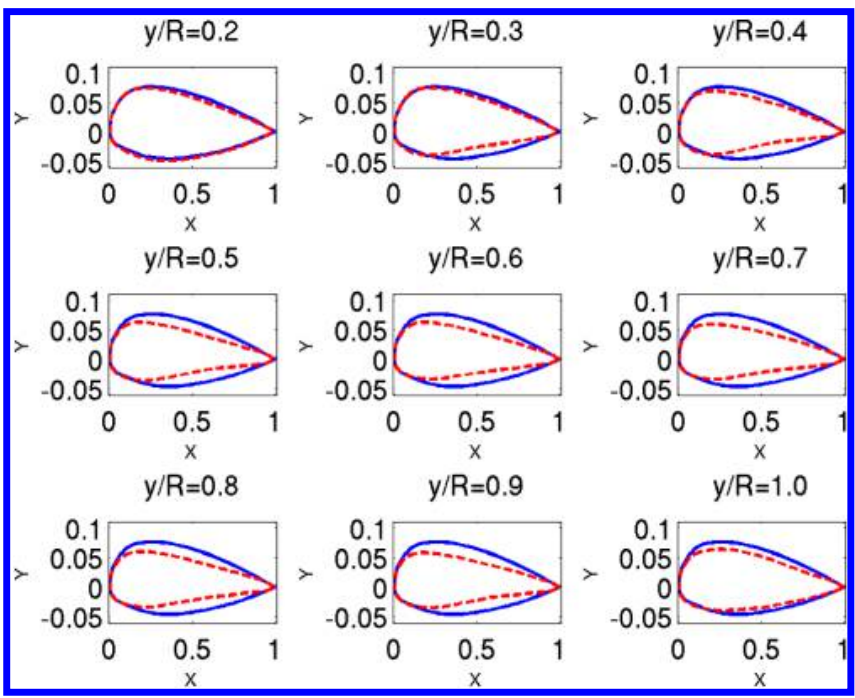

Fig. 15 HART-II rigid-blade optimized blade sections: baseline (solid lines), and optimized (dashed lines) [38]. in this figure and the subsequent figures are actually projected gradients, which are nothing but the norm of the projection of the negative gradient onto the bounding box defined by design variable bounds [36]. Figure $13 \mathrm{~b}$ further shows consistent convergence of all the three pitch parameters.

Figures 14 summarizes the optimized rotor blade shape as well as retrimmed rotor performance plots. Figure 14 a shows that the baseline trimmed rigid rotor is shape optimized to achieve approximately $4.7 \%$ power reduction with a penalty of approximately $2.3 \%$ thrust loss. Although some of this gain is lost upon retrim, the final optimized and trimmed rotor achieves an overall $2.8 \%$ reduction in power compared to the baseline blade. The near-identical lines of the baseline and retrimmed rotor thrust plots in Fig. 14b confirm that the rotor is retrimmed back to the trim target thrust value. Figure $14 \mathrm{c}$ shows a two-order-of-magnitude gradient drop and consistent functional convergence after 35 design iterations for the blade shape optimization. Figure 15 shows the shape-optimized blade sections at nine stations [38]. The optimized blade shape results in thicker inboard and thinner outboard stations.

Figures 16 illustrates the trim optimization of the flexible HART-II rotor to the trim target thrust $\left(C_{T}=0.0044\right)$ and moment values $\left(C_{M_{Y}}\right.$, $\left.C_{M_{Y}}=0\right)$. Figure $17 \mathrm{a}$ shows that the trim objective gradient drops by more than two orders of magnitude, whereas the objective functional achieves a minimum over 19 design iterations. Figure $17 \mathrm{~b}$ further shows consistent convergence of all the three pitch parameters.

Figure 18 summarizes the flexible-blade shape optimization, as well as retrimmed performance plots. Figure 18a shows the baseline trimmed flexible rotor is shape optimized to achieve a significant power reduction of approximately $5.0 \%$ with a thrust loss of approximately $2.6 \%$. Similar to the rigid-blade retrim, for the flexible blade, some of this gain is lost upon retrim. However, the final shapeoptimized and retrimmed rotor achieves an overall $3.1 \%$ reduction in

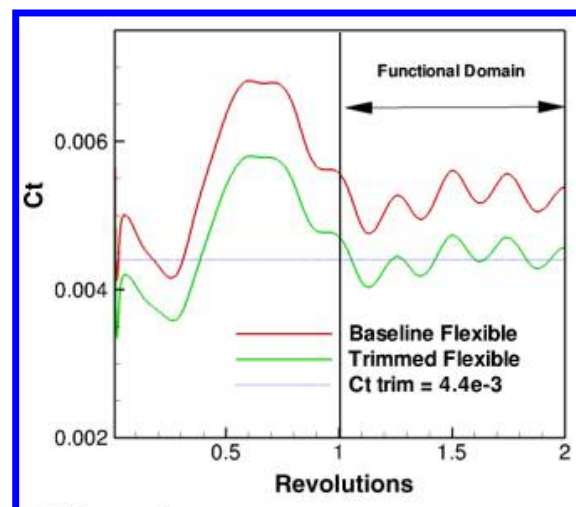

a) Thrust trim

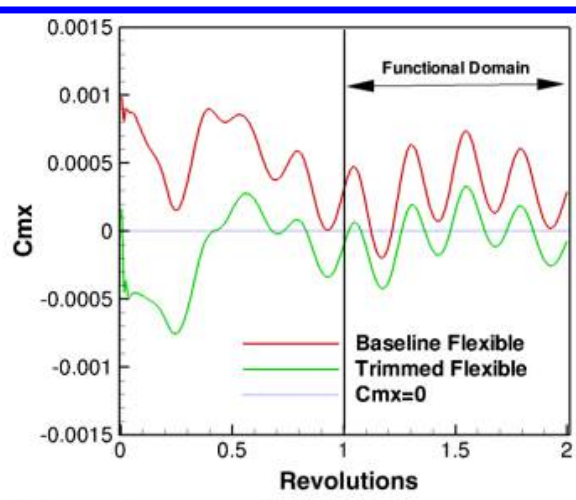

b) Lateral moment trim

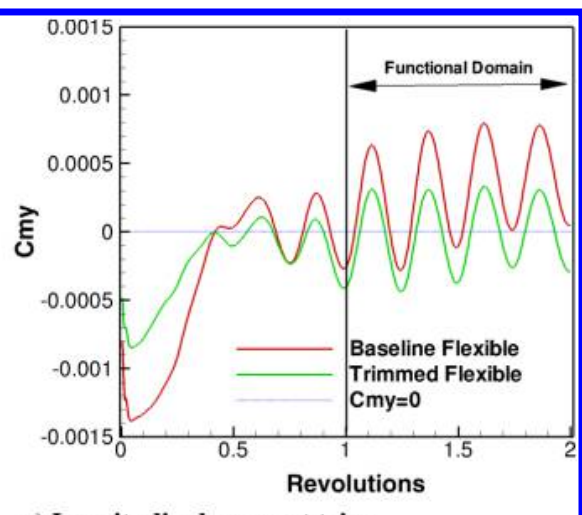

c) Longitudinal moment trim 


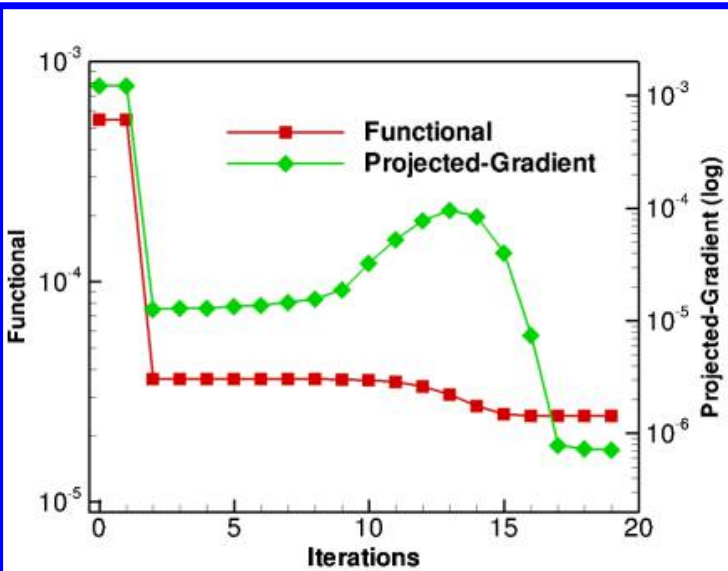

a) Trim optimization convergence

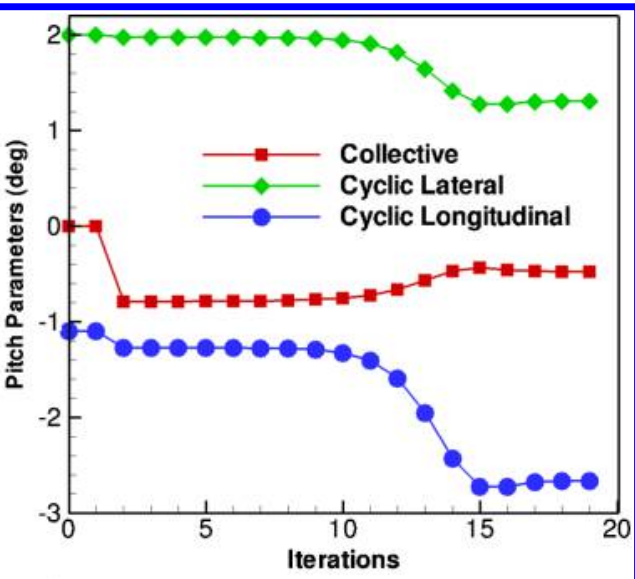

b) Pitch parameters convergence

Fig. 17 HART-II flexible-blade trim convergence.

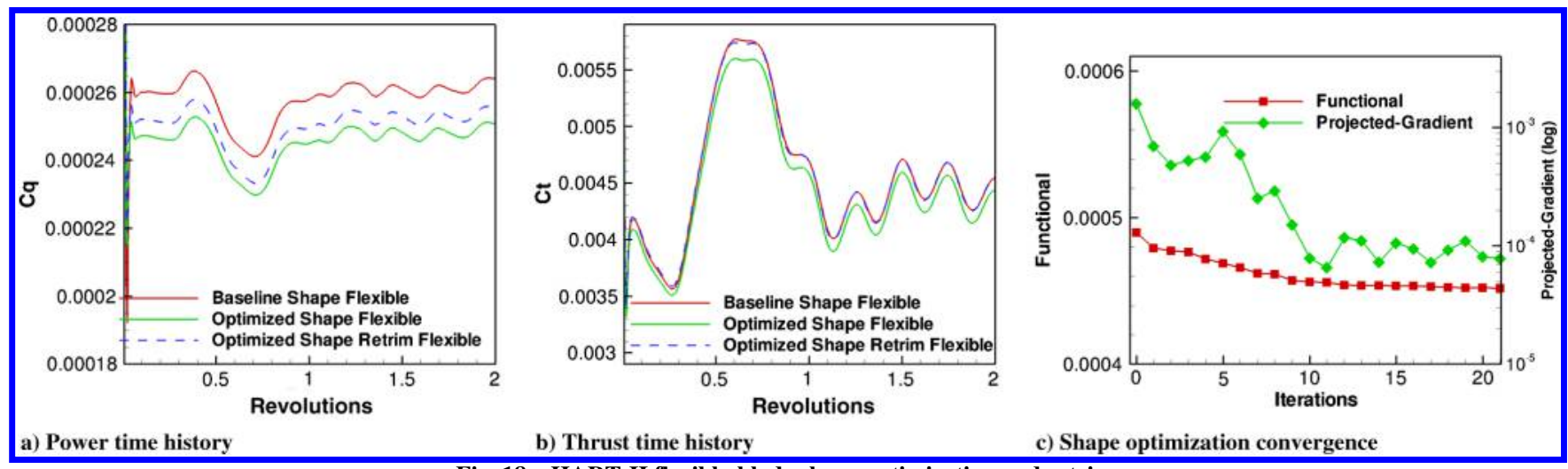

Fig. 18 HART-II flexible-blade shape optimization and retrim.

power compared to the baseline blade. Figure $18 \mathrm{c}$ shows more than one order of magnitude gradient drop and consistent functional convergence over 21 design iterations for the blade shape-optimization stage. Figure 19 shows the shape-optimized blade sections at nine stations. The optimized blade shapes show similar trends to those observed in the rigid-blade shape optimization, i.e., thicker inboard and thinner outboard stations.
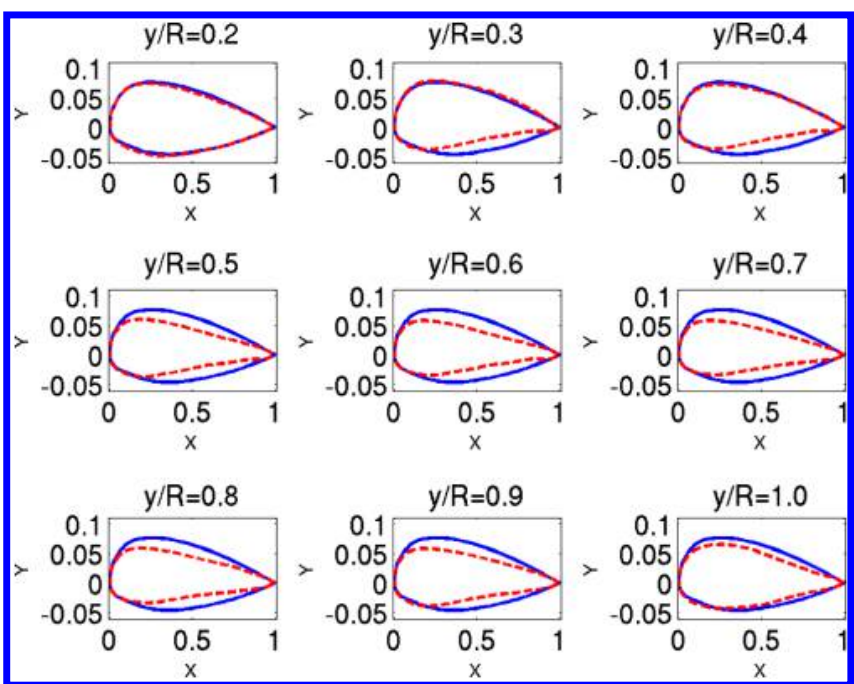

Fig. 19 HART-II flexible-blade optimized blade sections: baseline (solid lines), and optimized (dashed lines) [38].

\section{Conclusions}

In this work, a discrete adjoint formulation for time-dependent tightly coupled aeroelastic three-dimensional problems of flexible rotors in forward-flight conditions has been developed. The unsteady adjoint sensitivities of the formulation over several time steps have been verified, and the agreement was found to be sustained over a large number of time steps. The current formulation for rotor problems in forward-flight conditions was built upon a previously developed adjoint formulation for rotor problems in hover conditions by incorporating a blade cyclic pitch capability in the analysis formulation as well as in tangent and adjoint sensitivity analysis formulations. The formulation was designed to reuse, as much as possible, the original coupled aeroelastic data structures and solution strategies employed for the analysis problem, thus simplifying implementation and verification.

After successful verification of the developed adjoint optimization tool, it was effectively used to perform efficient rotor blade shape design targeting minimum rotor torque with the constraint of a prescribed rotor trim state. The performance optimization on both rigid and flexible blades consistently resulted in approximately $3.0 \%$ power reduction over the baseline blade shape while sustaining a target trim state.

\section{Acknowledgments}

This work was partly funded by the Alfred Gessow Rotorcraft Center of Excellence through a subcontract with the University of Maryland. Computer resources were provided by the University of Wyoming Advanced Research Computing Center and by the NCARWyoming Supercomputer Center. 


\section{References}

[1] Jameson, A., "Aerodynamic Shape Optimization Using the Adjoint Method," VKI Lecture Series on Aerodynamic Drag Prediction and Reduction, von Karman Inst. of Fluid Dynamics, Rhode St Genese, Belgium, 2003.

[2] Jameson, A., and Vassberg, J., "Computational Fluid Dynamics for Aerodynamic Design: Its Current and Future Impact," Proceedings of the 39th Aerospace Sciences Meeting and Exhibit, AIAA Paper 20010538, 2001. doi:10.2514/6.2001-538

[3] Nielsen, E., and Anderson, W., "Recent Improvements in Aerodynamic Design Optimization on Unstructured Meshes," AIAA Journal, Vol. 40, No. 6, 2002, pp. 1155-1163. doi: $10.2514 / 2.1765$

[4] Kim, S., Alonso, J., and Jameson, A., "Multi-Element High-Lift Configuration Design Optimization Using Viscous Continuous Adjoint Method," Journal of Aircraft, Vol. 41, No. 5, 2004, pp. 1082-1097. doi:10.2514/1.17

- [5] Giles, M., Duta, M., Muller, J., and Pierce, N. A., "Algorithm Developments for Discrete Adjoint Methods," AIAA Journal, Vol. 41, No. 2, 2003, pp. 198-205. doi: $10.2514 / 2.1961$

-[6] Economon, T. D., Palacios, F., and Alonso, J. J., "A Coupled-Adjoint Method for Aerodynamic and Aeroacoustic Optimization," 14th AIAA/ ISSM Multidisciplinary Analysis and Optimization Conference, AIAA Paper 2012-5598, 2012. doi:10.2514/6.2012-5598

-[7] Vassberg, J. C., Harrison, N. A., Roman, D. L., and Jameson, A., "A Systematic Study on the Impact of Dimensionality for a TwoDimensional Aerodynamic Optimization Model Problem," 29th AIAA Applied Aerodynamics Conference, AIAA Paper 2011-3176, 2011. doi:10.2514/6.2011-3176

-[8] LeDoux, S. T., Vassberg, J. C., Young, D. P., Fugal, S., Kamenetskiy, D., Huffman, W. P., Melvin, R. G., and Smith, M. F., "Study Based on the AIAA Aerodynamic Design Optimization Discussion Group Test Cases," AIAA Journal, Vol. 53, No. 7, 2015, pp. 1910-1935. doi:10.2514/1.J053535

-[9] Mavriplis, D. J., "Solution of the Unsteady Discrete Adjoint for ThreeDimensional Problems on Dynamically Deforming Unstructured Meshes," 46th Aerospace Sciences Meeting and Exhibit, AIAA Paper 2008-0727, 2008. doi: $10.2514 / 6.2008-727$

[10] Nielsen, E. J., Diskin, B., and Yamaleev, N., "Discrete Adjoint-Based Design Optimization of Unsteady Turbulent Flows on Dynamic Unstructured Grids," AIAA Journal, Vol. 48, No. 6, 2010, pp. 1195-1206. doi:10.2514/1.J050035

[11] Nielsen, E. J., Lee-Rausch, E. M., and Jones, W. T., "Adjoint-Based Design of Rotors in a Noninertial Reference Frame," Journal of Aircraft, Vol. 47, No. 2, 2010, pp. 638-646. doi: $10.2514 / 1.46044$

[12] Lee, S. W., and Kwon, O. J., "Aerodynamic Shape Optimization of Hovering Rotor Blades in Transonic Flow Using Unstructured Meshes," AIAA Journal, Vol. 44, No. 8, 2006, pp. 1816-1825. doi:10.2514/1.15385

[13] Mani, K., and Mavriplis, D. J., "Geometry Optimization in ThreeDimensional Unsteady Flow Problems Using the Discrete Adjoint," 51 st AIAA Aerospace Sciences Meeting, AIAA Paper 2013-0662, 2013. doi:10.2514/6.2013-662

[14] Dumont, A., Le Pape, A., Peter, J., and Huberson, S., "Aerodynamic Shape Optimization of Hovering Rotors Using a Discrete Adjoint of the Reynolds-Averaged Navier Stokes Equations," Journal of the American Helicopter Society, Vol. 56, No. 3, 2011, pp. 1-11. doi:10.4050/JAHS.56.032002

[15] Choi, S., Lee, K., Potsdam, M., and Alonso, J., "Helicopter Rotor Design Using a Time-Spectral and Adjoint-Based Method," Journal of Aircraft, Vol. 51, No. 2, 2014, pp. 412-423. doi:10.2514/1.C031975

[16] Martins, J. R. R. A., and Lambe, A. B., "Multidisciplinary Design Optimization: A Survey of Architectures," AIAA Journal, Vol. 51, No. 9 , 2013, pp. 2049-2075. doi:10.2514/1.J051895

[17] Kenway, G. K. W., Kennedy, G. J., and Martins, J. R. R. A., "Scalable Parallel Approach for High-Fidelity Steady-State Aeroelastic Analysis and Adjoint Derivative Computations," AIAA Journal, Vol. 52, No. 5, 2014, pp. 935-951. doi:10.2514/1.J052255

[18] Mishra, A., Mani, K., Mavriplis, D. J., and Sitaraman, J., "TimeDependent Adjoint-Based Optimization for Coupled Aeroelastic
Problems,” 21 st AIAA CFD Conference, AIAA Paper 2013-2906, 2013. doi: 10.2514/6.2013-2906

[19] Mishra, A., Mani, K., Mavriplis, D. J., and Sitaraman, J., "TimeDependent Adjoint-Based Aerodynamic Shape Optimization Applied to Helicopter Rotors," 70th American Helicopter Society Annual Forum, American Helicopter Soc. Paper 70-2014-0011, Montreal, 2014.

[20] Mishra, A., Mani, K., Mavriplis, D. J., and Sitaraman, J., "TimeDependent Adjoint-Based Aerodynamic Shape Optimization Applied to Helicopter Rotors," Journal of Computational Physics, Vol. 292, No. 1, 2015, pp. 253-271. doi:10.1016/j.jcp.2015.03.010

[21] Hodges, D. H., and Dowell, E. H., "Nonlinear Equations of Motion for the Elastic Bending and Torsion of Twisted Nonuniform Rotor Blades," NASA Ames Research Center and Army Air Mobility Research and Development Lab. Rept. NASA-TN-D-7818, Moffett Field, CA, 1974.

[22] Mavriplis, D. J., "Discrete Adjoint-Based Approach for Optimization Problems on Three-Dimensional Unstructured Meshes," AIAA Journal, Vol. 45, No. 4, 2007, pp. 741-750. doi: $10.2514 / 1.22743$

[23] Spalart, P. R., and Allmaras, S. R., "A One-Equation Turbulence Model for Aerodynamic Flows," La Recherche Aérospatiale, No. 1, 1994, pp. 5-21.

[24] Mavriplis, D. J., "Multigrid Strategies for Viscous Flow Solvers on Anisotropic Unstructured Meshes," Journal of Computational Physics, Vol. 145, No. 1, 1998, pp. 141-165. doi:10.1006/jcph.1998.6036

[25] Mavriplis, D. J., Yang, Z., and Long, M., "Results Using NSU3D for the First Aeroelastic Prediction Workshop," 51st Aerospace Sciences Meeting and Exhibit, AIAA Paper 2013-0786, 2013. doi:10.2514/6.2013-786

[26] Schuster, D. M., Chwalowski, P., Heeg, J., and Wieseman, C. D., "Summary of Data and Findings from the First Aeroelastic Prediction Workshop," 7th International Conference on Computational Fluid Dynamics (ICCFD7), International Conference on Computational Fluid Dynamics Paper ICCFD7-3101, Big Island, HI, 2012.

[27] Mishra, A., Mani, K., Mavriplis, D. J., and Sitaraman, J., "Helicopter Rotor Design Using Adjoint-Based Optimization in a Coupled CFDCSD Framework," 69th American Helicopter Society Annual Forum, American Helicopter Soc. Paper 69-2013-122, Phoenix, AZ, 2013.

[28] Yu, Y. H., Tung, C., van der Wall, B., Pausder, H.-J., Burley, C., Brooks, T., Beaumier, P., Delrieux, Y., Mercker, E., and Pengel, K., "The HARTII Test: Rotor Wakes and Aeroacoustics with Higher-Harmonic Pitch Control (HHC) Inputs -The Joint German/French/Dutch/US Project-," 58th American Helicopter Society Annual Forum, American Helicopter Soc. Paper 58-2002-00084, Montreal, 2002.

[29] Bir, G. S., Chopra, I., and Nguyen, K., "Development of UMARC (University of Maryland Advanced Rotorcraft Code)," 46th American Helicopter Society Annual Forum, American Helicopter Soc. Paper 46_1_004, Washington D.C., May 1990.

[30] Heckmann, A., Otter, M., Dietz, S., and López, J. D., "The DLR FlexibleBodies Library to Model Large Motions of Beams and of Flexible Bodies Exported from Finite Element Programs," 5th International Modelica Conference, Wien, Sept. 2006, pp. 85-95.

[31] Yang, Z., and Mavriplis, D. J., "A Mesh Deformation Strategy Optimized by the Adjoint Method on Unstructured Meshes," AIAA Journal, Vol. 45, No. 12, 2007, pp. 2885-2896. doi: $10.2514 / 1.30592$

[32] Mavriplis, D. J., "Time Dependent Adjoint Methods for Single and Multi-Disciplinary Problems," 38th VKI Lecture Series on Advanced Computational Fluid Dynamics: Adjoint Methods and Their Application in Computational Fluid Dynamics, von Karman Inst. of Fluid Dynamics, Rhode St Genese, Belgium, 2015.

[33] Nielsen, E. J., and Kleb, W. L., "Efficient Construction of Discrete Adjoint Operators on Unstructured Grids by Using Complex Variables," AIAA Journal, Vol. 44, No. 4, 2006, pp. 827-836. doi:10.2514/1.15830

[34] Nielsen, E. J., and Diskin, B., "Discrete Adjoint-Based Design for Unsteady Turbulent Flows on Dynamic Overset Unstructured Grids," AIAA Journal, Vol. 51, No. 6, 2013, pp. 1355-1373. doi:10.2514/1.J051859

[35] Nielsen, E. J., Lu, J., Park, M. A., and Darmofal, D. L., "An Implicit, Exact Dual Adjoint Solution Method for Turbulent Flows on Unstructured Grids," Computers and Fluids, Vol. 33, No. 9, 2004, pp. 1131-1155. doi:10.1016/j.compfluid.2003.09.005

[36] Zhu, C., Byrd, R. H., and Nocedal, J., "L-BFGS-B: Algorithm 778: LBFGS-B, FORTRAN Routines for Large Scale Bound Constrained Optimization," ACM Transactions on Mathematical Software, Vol. 23, 
No. 4,1997 , pp. $550-560$.

doi: $10.1145 / 279232.279236$

[37] Hicks, R., and Henne, P., "Wing Design by Numerical Optimization," Journal of Aircraft, Vol. 15, No. 7, 1978, pp. 407-412. doi: $10.2514 / 3.58379$

[38] Mishra, A., Mavriplis, D. J., and Sitaraman, J., “Time-Dependent Aeroelastic Adjoint-Based Aerodynamic Shape Optimization of Helicopter
Rotors in Forward Flight," 53rd AIAA Aerospace Sciences Meeting, AIAA Paper 2015-1130, 2015.

doi: $\underline{10.2514 / 6.2015-1130}$

M. Smith Associate Editor 
This article has been cited by:

1. Jan F. Kiviaho, Kevin Jacobson, Marilyn J. Smith, Graeme Kennedy. A Robust and Flexible Coupling Framework for Aeroelastic Analysis and Optimization . [Citation] [PDF] [PDF Plus] 